\title{
JUDO: Just-in-time rumour detection in streaming social platforms
}

\author{
Thanh Toan Nguyen ${ }^{\mathrm{a}}$, Thanh Tam Nguyen ${ }^{\mathrm{b}}$, Thanh Thi Nguyen ${ }^{\mathrm{c}}$, Bay Vo ${ }^{\mathrm{b}}$, \\ Jun $\mathrm{Jo}^{\mathrm{a}}$, Quoc Viet Hung Nguyen ${ }^{\mathrm{a}}$ \\ ${ }^{a}$ Griffith University, Australia \\ ${ }^{b}$ Faculty of Information Technology, Ho Chi Minh City University of Technology \\ (HUTECH), Ho Chi Minh City, Vietnam \\ ${ }^{c}$ Deakin University, Australia
}

\begin{abstract}
Web platforms, especially social media, are facing a new and ever-evolving cyber threat operating at the information level. Their open nature allows a high velocity flow of rumours that emerge unexpectedly and spread quickly. While rumour detection has attracted many theoretical and practice studies, the timing of the detection is often neglected or not properly considered. Rumours often cause irreversible damage worldwide before being successfully detected. To address this, we approach early rumour detection from a streaming perspective. We present a just-in-time rumour detection framework that is built on top of the continuous scoring of rumour-related signals. To overcome the trade-off between timeliness and the coefficient of detection, our model treats social graphs as a data stream and computes the anomaly score of potential rumours at both the element-level and subgraph-level. This multi-level approach not only captures the propagation structure of rumours but also focuses on abnormal elements that are responsible for bootstrapping or amplifying the rumours (the 'explore vs exploit' effect). With extensive evaluations on our published benchmark, our model identifies rumours earlier than the baselines while achieving an even better detection coefficient.
\end{abstract}

Keywords: rumour detection, anomaly scoring, streaming social data

Preprint submitted to Information Sciences

June 6, 2021 


\section{Introduction}

While the Web has been a target for sophisticated cyberattacks at the hardware and software levels, it is facing a new and ever-evolving cyber threat operating at the information level [1]. Moving toward globalisation, the social media 5 ecosystem is going through a transition phase that eases access to topical issues but tends to amplify rumours, consequently undermining the public's trust in the media. Rumours could arise from several levels of intentions, ranging from 'honest' journalists' negligence to large-scale orchestrated campaigns. Assuming malicious intent, the objectives could be financial, through the creation of artificially shocking content to maximise advertisement revenue (e.g., click bait), or political, by favouring a political party to influence votes. Developing techniques to understand and mitigate the impact of rumours is, therefore, of tremendous importance.

Since the open nature of social platforms allows a massive and continuous flow of unverified information, rumours emerge unexpectedly and spread quickly. More than often, rumours make a severe impact worldwide before being detected [2, 3. As a result, state-of-the-art surveys in the literature are calling for early rumour detection [4, [5]. This paradigm has attracted a lot of attention; in particular, there has been research on sequential models such as recurrent neural networks and long short-term memory (LSTM) 6].

However, there are still severe limitations that have not been solved completely [7. First, the detection techniques assume a fixed structure of rumour propagation. In particular, they are often based on a sequence of retweet cascades from an original post [8]. However, rumours can come from different sources and spread via the use of hashtags or suspicious links rather than just retweets 9. Second, rumour propagation is not fully captured. Rumours are often classified as a fixed set or sequence of social entities [10] rather than having their structure revealed. Some works try to define a set of propagation patterns beforehand [11, 12. However, since rumours can propagate via 'innocent' users, who accidentally spread rumourous posts or have their accounts 
hacked, the propagation can be unexpected and may not follow a pre-defined pattern [7, 13]. Third, the detection results are not fully 'early'. For example, the classification-based approaches often need a pre-processed sequence of data to classify it as a rumour or not [6]. This supposition requires knowing when to cut the sequence for detection. If cutting is done too late, the rumours may have already caused irreversible damages. Otherwise, there might not be enough information for detection. This trade-off between the detection coefficient and the timeliness of detection is illustrated in Figure 1, where existing methods are compared with our method (i.e., JUDO). The goal of this paper is to go beyond this trade-off by achieving timely rumour detection with a decent detection coefficient. A more detailed empirical comparison will be presented in Section 9.

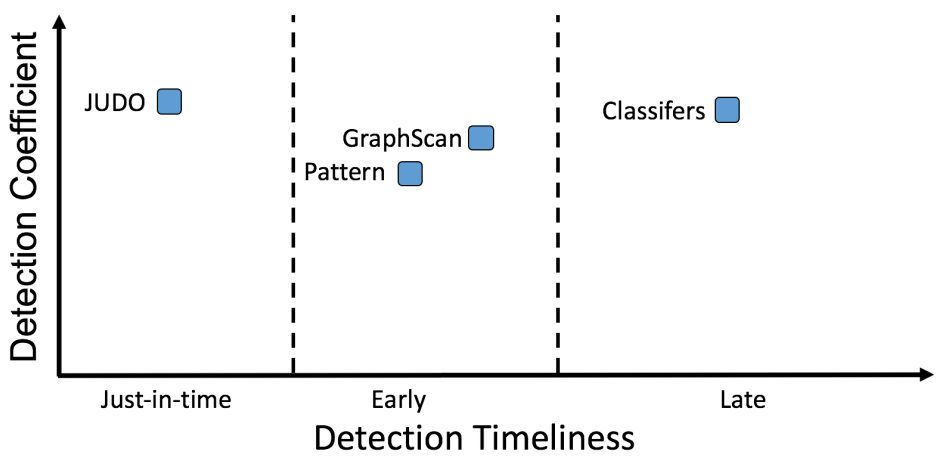

Figure 1: Trade-off between detection coefficient and detection timeliness.

In this paper, we propose a just-in-time rumour detection (JUDO) framework to overcome these limitations. The core idea of our framework is the combination of monitoring and detection paradigms, where potential rumours are scored by their anomaly signals. However, we go beyond a simple thresholding approach in several aspects. First, our approach is compatible with streaming data. This allows timely detection, since rumours are constantly monitored from their genesis. Second, our approach leverages the 'explore vs exploit' ef${ }_{50}$ fect, where social entities are scored not only individually but also collectively. This reduces the false accusation of rumour-related entities (false alarms), since 
suspicious entities are cross-checked by their connections. Third, the structure of rumour propagation is captured by a subgraph, a generic representation of social networks themselves. This allows the detection of rumours that spread not only via entities with high anomaly signals but also through normal entities.

Designing such a framework has several challenges. First, the anomaly scoring mechanism needs to be efficient with the arrival of new data while still providing sufficient information to assess the anomalousness of data effectively. Second, it needs to be compatible with different modalities of data such as users, posts, links, and hashtags, which do not share the same semantic space. Third, streaming data is usually not stationary in practice, i.e., a data item that is anomalous in the past might not be anomalous in the future. The scoring model needs to be adaptive to changes in data distributions.

With the goal of overcoming these challenges, our paper makes the following contributions along with its structure.

- Just-in-time problem: Section 4 formulates just-in-time rumour detection based on an anomaly scoring problem. Potential rumours can be monitored constantly and detected just-in-time when they emerge to become true rumours.

- First-order anomaly scoring: Section 5 develops an online algorithm for computing first-order anomaly signals. Going beyond the state of the art, we do not need to store all or a sequence of historical data. Instead, we develop a locality-sensitive hashing (LSH) based indexing structure that computes the anomaly score as the collision probability in constant time.

- High-order anomaly scoring: Section 6 develops an algorithm to score and extract high-order rumour structure. The scoring reflects the tradeoff between the explore aspect (includes as many entities affected by the rumour as possible) and the exploit aspect (favours propagation structure with a significant number of anomalous entities).

- End-to-end process: Section 7 puts the first-order and high-order anomaly 
signals together for an end-to-end anomaly scoring process. This process scores and detects a list of subgraphs that emerge as rumours.

- Adaptive process: Section 8 extends the end-to-end process with the ability to adapt to different conditions of streaming data such as concept drifts and the data input rate. This makes our framework robust to rumours of new domains in social media as well as in-time even when rumours propagate faster than before.

The remaining sections of the paper include the following: Section 2 discusses related work and Section 3 motivates the need to capture rumour propagation via anomaly signals such as inconsistency and abnormal values. Section 9 reports the empirical evaluations of our framework with real-world datasets, and Section 10 summarises the paper.

\section{Related work}

Social data representation. A graph is a natural representation of social platforms [14, 15, 16. While a uni-modal social graph models the relationships of entities of the same type, a multi-modal social graph captures the relationships of entities from different domains, such as a user and a post [17. Existing studies on rumour detection in social platforms focus on the propagation patterns of known events [10] or phenomena [4]. These settings are orthogonal to our work, as we strive to detect rumours by capturing and cross-checking the collective signals of emerging phenomena without any prior knowledge. Moreover, designing an efficient detection framework for large-scale networks is challenging [18, 19]; however, this is unavoidable for real-time social platform applications.

Early rumour detection. Most rumour detection techniques [4, 20, 3] do not fully support early detection, as they treat the problem as rumour classification for non-streaming data. Most related to our work is [5], which studies how much the delay in detection can be minimised. However, the authors solve an orthogonal problem, which computes the minimum number of posts required 
before an event is classified as a rumour. In our work, we fully support early rumour detection by (i) progressively monitoring and scoring rumour-related elements in the streaming data and (ii) capturing the rumour structures as maximal subgraphs. Recently, some techniques support (i) or (ii) but not both. One is based on anomaly detection [9] but needs the whole graph data as input. Another is based on pattern matching [11, 12, which requires a definition of pattern beforehand and cannot detect rumours with new patterns.

Streaming social signals. Research on monitoring social media data monitor rumourous content by integrating signals from multiple sources. This is done by associating each data entity with a rumour index, which reflects its current state of being influenced by harmful information. Measuring falsehood is essentially a problem of probability modelling, which compresses detective cues into a probability value, as studied in Bayesian statistics, frequentist statistics, and machine learning [1, 21]. Several detective cues have been investigated [4, 8, 22]: (1) Feature-based - quantifies writing styles, either manually defined by experts or automatically learned by AI deep learning methods; (2) User-based - studies how users engage with rumour posts and what roles users play in their creation and dissemination; (3) Propagation-based - models the cascade of rumours, e.g., using a tree-like structure rooted from the genesis post. However, existing techniques require a specific data entity as input. Otherwise, tracking all of them may result in isolated cues and few meaningful links.

Combining the best of these approaches, our work monitors social entities without storing their historical data. If they show anomaly signals for potential rumours, they are aggregated into connected subgraphs as a whole to represent the propagation structure of respective rumours.

\section{Problem setting}

This section introduces the correlation between rumours and anomaly signals in social media Section 3.1). Then, we discuss a motivating example of how to exploit anomaly signals for rumour detection in Section 3.2. Next, Section 3.3 
presents the relevant background that is necessary for understanding how to compute anomaly signals in a streaming setting.

\subsection{Rumour as anomaly signals}

Rumour is one of the anomalous phenomena in social platforms, and it often spreads undetected at the time of posting [23]. Rumours are sets of anomaly signals that are in relationships with each other (i.e., users, posts, links, hashtags) [9].

In this study, we focus on anomaly signals that support rumour detection. Many researchers study various anomaly signals by computing the statistical scores of entities, such as posts and users [24]. However, statistical scores do not preserve graph structures [25]. Instead, our model designs a statistical structure that preserves first-order and high-order anomaly signals. This is because rumours are often propagated in a channelled manner, in which the entities related to a rumour are anomalous [2]. Existing statistical scoring methods are often applied to a single modality, while our proposed model is applicable for multiple modalities including users, posts, links, likes, hashtags, and their interactions.

\subsection{Anomaly signals for rumour detection}

We approach the rumour detection problem using first-order and high-order inconsistency signals. Figure 2 illustrates a generic social graph with four types of entities: users, posts, links, and hashtags. Each entity is associated with a vector of features. An edge between two entities represents a relation, such as a post containing a link. Specifically, in our model, each relation is also associated with a vector of features such as the time difference of appearance between two entities.

First-order signals. Anomaly signals for rumours are often disclosed in inconsistencies between entities and relations. For instance, in Figure 2, we can see that User 2 has an abnormal registration age and an abnormal number of 


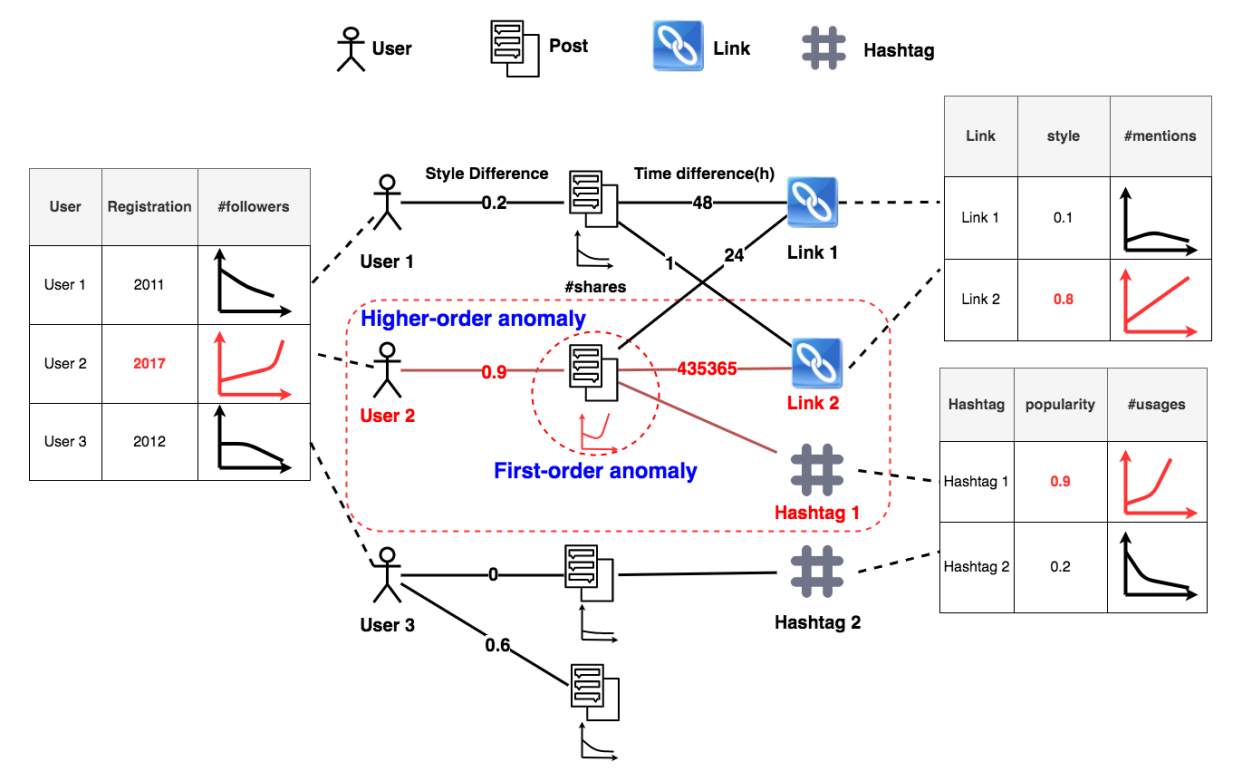

Figure 2: Rumour detection from anomaly signals.

followers compared to others. Other highlighted entities and relations also have abnormal features.

High-order signals. However, these first-order signals are inadequate and unreliable. For example, a hype from a user's post also increases the number of followers. To achieve a higher confidence, we use high-order signals to incorporate first-order signals with their spatial relation to confirm the more compact and global anomalies. In other word, rumours in the social platform are expected to occur at a structural level rather than an entity level. Going beyond traditional entity settings, our approach further includes the relations in anomaly signals. Taking Figure 2 for instance, the difference between the posting time of Link 2 and the genesis time of the post is abnormally large, indicating that the posting user uses false evidence.

Although this example uses entity names and feature names quite similar to Twitter, our framework is generic for other platforms [26] due to the common graph representation Section 4). Based on this representation, we compute the first-order anomaly signals of all incoming data elements in a streaming social 
network. Then, a high-order scoring component is incorporated to eliminate the false positives by cross-checking first-order signals and validating the results.

\subsection{Computing streaming signals}

Computing statistical scores in the high-velocity data streams of social platforms is challenging. Typically, statistical scoring is applied to the whole or a sample of data [25. This luxury is not suitable for rumour detection since rumours often cause irreversible damage when they are detected too late [2].

To overcome this challenge, we leverage the collision property of LocalitySensitive Hashing (LSH) [27] to compute signals. As a reminder, LSH is a hashbased indexing technique sacrificing some degrees of collision for speed. Among many other LSH implementations [28, Signed Random Projections (SRP) 29] is the most popular algorithm; we used it heavily in this work. However, it is worth noting that our proposed framework can naturally be adapted with any feasible alternative classes of the LSH family.

Our work is pioneering in designing LSH-based anomaly signals for rumour detection. While the collision cases in LSH are often regarded as 'bad' in the literature, we leverage them to compute anomaly signals incrementally in fast streaming data. The core idea is that anomaly signals are often significantly different from normal values. As a result, if a data value has a small probability of collision, it is likely to be an anomaly signal.

\section{Problem definition}

Let us begin with formally defining the problem of anomaly scoring through multi-modal social graphs. Compared to traditional rumour detection, which learns a label function of entities (as either a rumour or not), our goal is to learn a scoring function of entities that is updated for every arrival of new data. In other words, we monitor the anomaly signals of social entities and let the decision-makers decide to report the most anomalous ones. To this end, it is desirable first to have a unified representation to encode different types of social entities. 
Definition 1 (Multi-modal social networks) Multi-modal social networks (or social graphs) are defined as an undirected graph $\mathcal{G}=(V, E, \vartheta, \varepsilon)$ where $V=\left\{V_{1} \cup \ldots \cup V_{C}\right\}, V_{c}$ refers to the set of entity type $c, C$ is the total number of entity types, $E=\left\{E_{1} \cup \ldots \cup E_{Q}\right\}, E_{q}$ refers to the set of relation type $q, Q$ is the total number of relation types, $\vartheta=\left\{\vartheta_{1} \ldots \vartheta_{C}\right\}$ is a set of $C$ mapping functions, $\vartheta_{c}: V \rightarrow \mathbb{R}^{d_{c}}$ defines a $d_{c}$-dimensional feature vector $\vartheta_{c}(v)$ for each node $v$ of type $c, \varepsilon=\left\{\varepsilon_{1} \ldots \varepsilon_{Q}\right\}$ is a set of $Q$ mapping functions, $\varepsilon_{q}: E \rightarrow \mathbb{R}^{d_{q}}$ defines a $d_{q}$-dimensional feature vector $\varepsilon_{q}(e)$ for each edge e of type $q$.

Informally, we model a social platform as a multi-modal social graph where 220 each node is an entity, such as users, tweets, links, and hashtags, and each edge represents a relation between entities. We call an entity or a relation as an element of the social graph. Each element is associated with a feature vector whose dimension may vary based on its modality. For instance, in Figure 2 , each tweet has only one feature while each user has two features. Besides entities themselves, the propagation of rumours could be captured by the relations between entities.

Definition 2 (First-order anomaly signals) An anomaly graph of a social graph $\mathcal{G}=(V, E, \vartheta, \varepsilon)$ is defined as an undirected graph $\mathcal{A}=\left(V, E, \alpha_{v}, \alpha_{e}\right)$ where $\alpha_{v}: V \rightarrow[0,1]$ and $\alpha_{e}: E \rightarrow[0,1]$ are mapping functions that assign anomaly scores to entities and relations, respectively.

For consistency, our scoring convention is that the larger an anomaly score is, the more likely it is that it indicates a rumour.

Example 1 In Figure 3, after computing the first-order anomaly scores for each entity and relation, we have an anomaly graph $\mathcal{A}=\left(V, E, \alpha_{v}, \alpha_{e}\right)$, where $V=$ ${ }_{235}\left\{v_{1}, v_{2}, \ldots\right\}, E=\left\{e_{1}, e_{2}, \ldots\right\}, \alpha_{v}=\left\{v_{1} \rightarrow 0.95, \ldots\right\}, \alpha_{e}=\left\{e_{1} \rightarrow 0.15, \ldots\right\}$.

Definition 3 (High-order anomaly signals) A high-order anomaly graph of a social graph $\mathcal{G}=(V, E, \vartheta, \varepsilon)$ is defined as $\mathcal{H}=\left(\mathbb{S}, \alpha_{s}\right)$, where each element $S \in \mathbb{S}$ is a subgraph ( $S \subset V \cup E$ and $S$ is connected) and $\alpha_{s}: \mathbb{S} \rightarrow[0,1]$ is a scoring function that evaluates the anomalousness of a subgraph. 


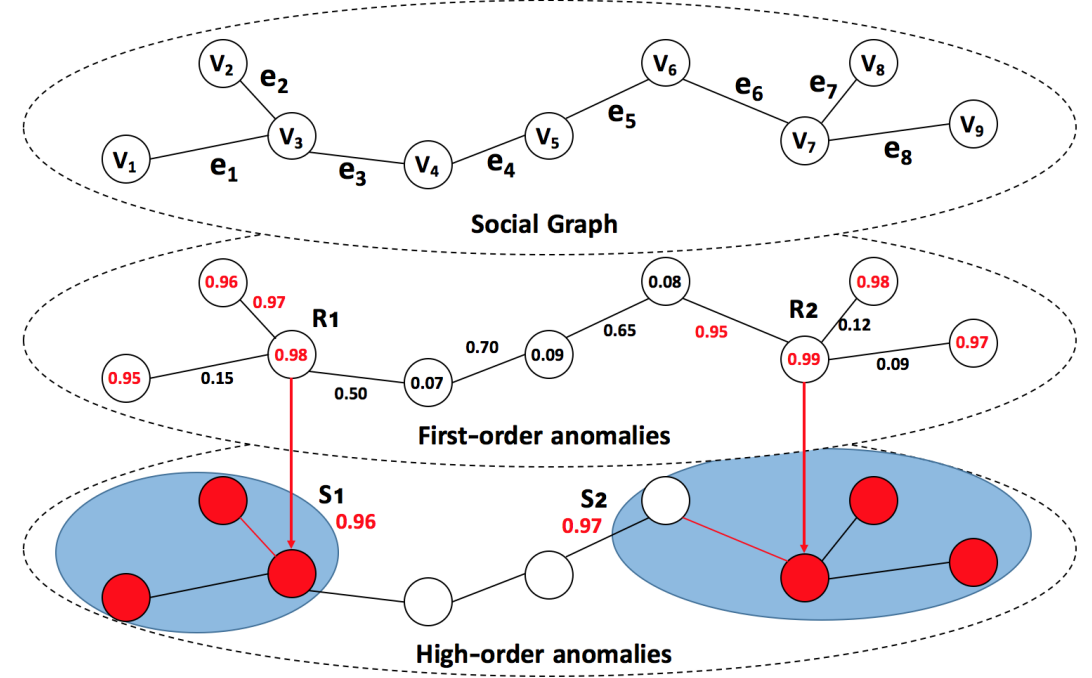

Figure 3: First-order and high-order anomaly signals.

Due to its propagation behaviour, a rumour is often a subgraph consisting of entities and relations. An explicit definition of how much anomalous this subgraph is and how many elements it contains is unknown, so that we rely on anomaly scoring. That is, the anomaly scores of rumour elements are significantly larger than those of non-rumour elements. Our goal is to design a scoring function:

Definition 4 (Anomaly scoring) Given a social graph $\mathcal{G}=(V, E, \vartheta, \varepsilon)$, the problem of anomaly scoring is to retrieve a ranked list $\mathcal{R}=\left(R_{1}, \ldots, R_{k}\right)$, where $R_{i} \subseteq V \cup E$, such that the closer $R_{i}$ is to the top of the list, the larger the first-order and high-order anomaly scores it has (and thus, the more likely it is that it is a true rumour).

Design Principles. Anomaly scoring in streaming social media needs to overcome the trade-off between the explore and exploit effects [30]. While traditional anomaly scoring relies on the whole or a sample of data to compute statistical signals, doing so in streaming social media induces an overhead of recomputation, which delays the detection of rumours for damage mitigation. Besides this 
Table 1: Notation summary.

\begin{tabular}{ll}
\hline Notation & Definition \\
\hline $\mathcal{G}$ & multi-modal social graph \\
$\mathcal{A}$ & first-order anomaly graph \\
$\mathcal{H}$ & high-order anomaly graph \\
$\widehat{F}\left(v_{i}\right)$ & first-order anomaly score of $v_{i}$ \\
$\widehat{H}(S)$ & high-order anomaly score of subgraph $S$ \\
$\mathcal{R}$ & a list of rumour structures \\
\hline
\end{tabular}

trade-off, we argue that a complete solution should also satisfy the following principles:

(R1) Multi-modal: Anomaly signals can come from many different modalities. This calls for a unified anomaly scoring model for entities or relations from different modalities and feature domains.

(R2) Non-parametric: The detection model should compute anomaly signals without any expectation about the baseline distribution. As a result, threshold-based anomaly detection techniques are not applicable since the characteristics of the data distribution cannot be estimated. This also requires the solution to be independent of the feature domain and value ordering.

(R3) Adaptive to emergent rumours: The scoring model should cover not only long-term history information for anomaly detection but also adapt quickly to new short-term rumours, which may not be significantly anomalous compared to regular data.

Table 1 summarises the notations that are frequently used in this paper.

\section{First-order anomaly scoring}

This section implements the first-order anomaly scoring. First, we design features related to rumours Section 5.1). Then, we reveal the indexing mecha- 
local anomaly indexing structure (Section 5.3). Based on this, a final algorithm for first-order rumour detection is provided (Section 5.4).

\subsection{Anomaly features}

From our various studies [9, we come up with a list of the most representative features in Table 2. These features are applicable for various social platforms, although their names may be different in a particular platform.

- Post: An abnormal post can be a post with a high number of shares, high usage of provoking keywords, and biased linguistic style.

- User: We look for anomaly signals such as registration age, status frequency, \#friends/followers, and \#posts. For example, an abnormal user could be the one who registered just a few days ago but has an abnormally high number of posts and friends/followers. It could indicate these accounts are created to spread rumours and hide true identities.

- Link: By link, we mean the article linked in a post. An abnormal article may have a high number of mentions and biased linguistic style.

- Hashtag: Hashtags are also used to spread rumours. An abnormal hashtag may have a high number of usages and sudden changes in popularity.

- Post-Link: The relation between a post and a link can identify anomaly signals such as time difference, location difference, and style difference. Rumours may use inaccurate articles as evidence to support their claims.

- User-Post: The relation between a user and a post can also contain signals such as style difference. If a post has a linguistic style different from what the user normally uses, it may be the case that the user is spreading a rumour.

- User-Link: An anomaly happens when a user usually posting BBC News now suddenly refers to unofficial sources. This can be captured by the source difference in the relation between a user and a link.

- User-Hashtag: A new hashtag never used before by a user could indicate an abnormal behaviour. This is captured by the usage difference between 
hashtags the user normally uses and the given hashtag.

- Link-Hashtag: If a new hashtag is suddenly used excessively in a linked article, it may indicate that a rumour is emerging.

Table 2: First-order anomaly signals.

\begin{tabular}{|c|c|c|}
\hline & Element & Feature \\
\hline \multirow{4}{*}{ 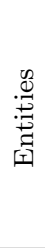 } & Post & \#shares, keywords, linguistic style \\
\hline & User & \#friends/followers, \#posts, registration age, status frequency \\
\hline & Link & \#mentions, linguistic style \\
\hline & Hashtag & \#usages, popularity \\
\hline \multirow{5}{*}{ 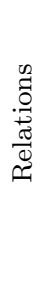 } & Post-Link & time difference, location difference, style difference \\
\hline & User-Post & style difference \\
\hline & User-Link & source difference \\
\hline & User-Hashtag & usage difference \\
\hline & Link-Hashtag & mention frequency \\
\hline
\end{tabular}

These features cover different modalities (R1) and boost the confidence of rumour detection as a whole. However, using them separately may lead to false positives. Below, we show how to combine these features simultaneously for anomaly scoring with high confidence.

\subsection{Indexing anomaly signals}

Here, we describe a streaming anomaly scoring model for incrementally computing the anomaly score for each incoming data item from the same type of entity or relation. For simplicity, we use entities from one type (i.e., the post type) to demonstrate how the data structure works. However, the same approach can be applied to other entity and relation types by maintaining each sketch structure for each data type.

LSH Reminder. LSH is a family of hash functions, which indexes data values into buckets with some probability of collision [27]. More precisely, we denote 
$\mathcal{H}$ as a family of hash functions that projects data items from $\mathbb{R}^{d}$ to a discrete set $[0, \mathcal{B}]$. Given an likeliness ratio $\omega$, a distance value $r$, and probability values $p_{1}$ and $p_{2}$, where $p_{1}>p_{2}$, a family of hash functions $\mathcal{F}=\left\{h: \mathbb{R}^{d} \rightarrow \mathcal{B}\right\}$ is considered $\left(r, \omega r, p_{1}, p_{2}\right)$-locality sensitive if with every two data items $d_{1}, d_{2} \in$ $325 \mathbb{R}^{d}$, it satisfies both of the following conditions:

1. If $\left\|d_{1}, d_{2}\right\| \leq r$ then $p\left[h\left(d_{1}\right)=h\left(d_{2}\right)\right] \geq p_{1}$

2. If $\left\|d_{1}, d_{2}\right\| \geq \omega r$ then $p\left[h\left(d_{1}\right)=h\left(d_{2}\right)\right] \leq p_{2}$

It is worth noting that the collision could occur when two data items are hashed into the same bucket, meaning that their hash values are equal (i.e., $h\left(d_{1}\right)=$ $\left.330 \quad h\left(d_{2}\right)\right)$.

Anomalies as rare collisions. Take a historical data $D_{t}=\left\{d_{1}, \ldots, d_{t}\right\}$ of a social element over stream, in which each $d_{i}$ is a vector of features 1..L. Anomalies are defined as data which is significantly separated from average data. In particular, in social data, the reasonable statistics of an anomaly element $d_{i}$ to all other $d_{j} \in D_{t}$ shall deviate significantly from common data elements. In an offline setting, there are methods to score the anomalousness by the distance from $d_{i}$ to all other elements $d_{j} \in V_{t}$ with reasonably good statistics [31. It is required for a complete scan over the dataset to compute the anomaly score of every single $d_{i}$ (denoted as $\widehat{F}\left(v_{i}\right)$ ).

Here, we employ the random projections with signed (SRP) 29, 32, which is a variant of the LSH family based on the cosine similarity distance. We choose SRP because of its popular and simplicity; however, the algorithm can naturally be adapted to work with any LSH classes. Given a vector $d_{i}$, SRP utilises an arbitrary window $w$ with each component randomly sampled from the normal distribution and solely retains the sign. More precisely, the projection function is formulated as:

$$
h_{w}\left(d_{i}\right)=\operatorname{sgn}\left(w^{T} d_{i}\right)
$$

More precisely, the function is a mapping $h_{w}\left(d_{i}\right): \mathbb{R}^{n} \rightarrow\{0,1\}$. The study 33 proved that the collision under such hashing satisfies the condition 
below:

$$
p\left(d_{i}, d_{j}\right)=p\left[h_{w}\left(d_{i}\right)=h_{w}\left(d_{j}\right)\right]=1-\frac{\lambda}{\pi},
$$

where $\lambda$ (i.e., the cosine similarity) is computed by:

$$
\lambda=\cos ^{-1}\left(\frac{d_{i}^{T} d_{j}}{\left\|d_{i}\right\|_{2} \cdot\left\|d_{j}\right\|_{2}}\right) \cdot \frac{d_{i}^{T} d_{j}}{\left\|d_{i}\right\|_{2} \cdot\left\|d_{j}\right\|_{2}}
$$

Meta-hash function. We define a meta-hash function $\mathcal{M}_{l}$ as a combination of $M$ independent hash functions by sampling $M$ independent windows $w$. More precisely, $\mathcal{M}_{l}=\left\{h_{l 1}, \ldots, h_{l M}\right\}$. While a single SRP hash function returns a onebit output, the meta-hash function yields an M-bit output $[\{0,1\}]^{M}$. Following this idea, the new collision probability when applying the meta-hash function is recomputed by this equation:

$$
p\left(d_{i}, d_{j}\right)=p\left[h_{w}\left(d_{i}\right)=h_{w}\left(d_{j}\right)\right]=\left(1-\frac{\lambda}{\pi}\right)^{M}
$$

The resulting collision probability decreases dramatically by the power law of probability, and this behaviour is further demonstrated in the experiment section. This evaluation does not assume any data distribution in advance, thus satisfying (R2).

\subsection{Online indexing of anomaly signals}

In the online setting, it is worth noting that the memory usage and update time are bounded. Thus, we strive for scoring functions that can be efficiently estimated on the fly. More precisely, any shift in data from $D_{t}$ to $D_{t^{\prime}>t}$ needs a mechanism to reflect the change immediately. As explained in the earlier section, scoring functions that favour these properties are formulated as:

$$
\widehat{F}\left(d_{i}\right)=\frac{1}{\left|D_{t}\right|} \sum_{d_{j} \in D_{t}} 1-p\left(d_{i}, d_{j}\right)^{M}
$$

where $p$ is the probability of collision and $M \geq 1$ is an integer representing for the number of projections. The intuition behind this is that the data item different from others in a data distribution (low collision probability) shall be 
highly anomalous. We will use this observation to detect anomalies efficiently, and the details of the data structure and algorithm will be presented below.

LSH-based data sketch. Similar to data counting sketches 34, 35, we design an LSH-based data sketch consisting of $L$ arrays for $L$ different meta-hash functions $\mathcal{M}=\left\{\mathcal{M}_{1}, \ldots, \mathcal{M}_{L}\right\}$, each of which is responsible for indexing the features of an entity in the social graph. Figure 4 presents the structure of the sketch. Different from the LSH family for nearest-neighbour search, which requires extra distance computation of the candidates, our scoring model just retrieves the counting. The process is extremely efficient, in terms of both memory usage and speed.

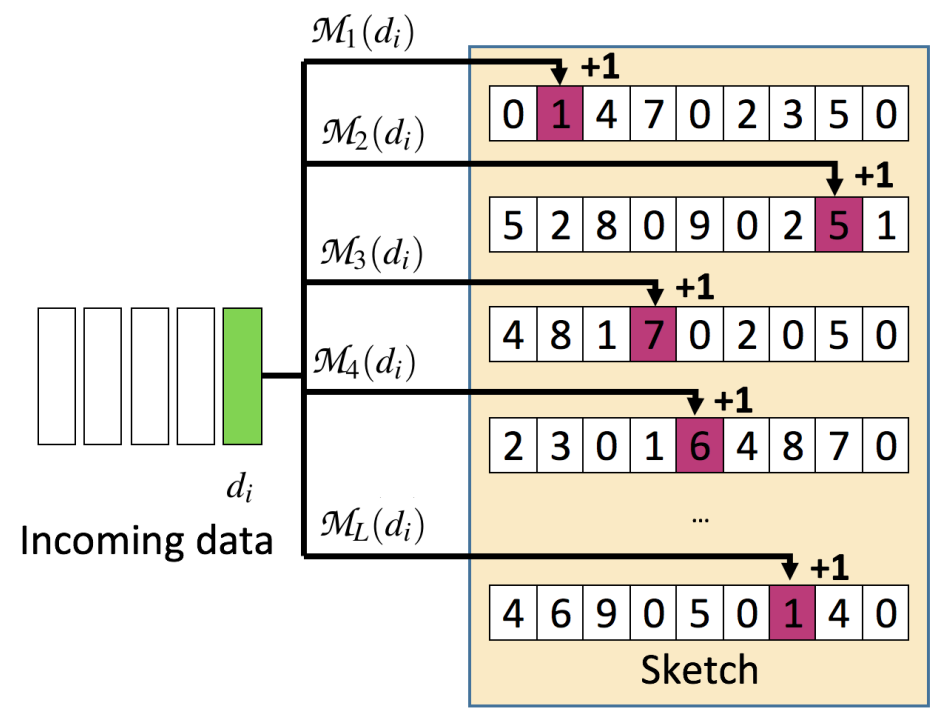

Figure 4: The data structure of the scoring model.

Example 2 Assume that a user has a streaming data of two features: number of friends and number of posts, as $\{10,11,10,11, \ldots, 1000\}$ and $\{3,4,4,3, \ldots, 69\}$. The first-order scores for this user at each time step are $\{0.0,0.002,0.003$, $0.007, \ldots, 0.981\}$, respectively. It can be seen that the user has two abnormal values (1000 and 69) in the end; thus, his anomaly score at that time is very high (0.981). 


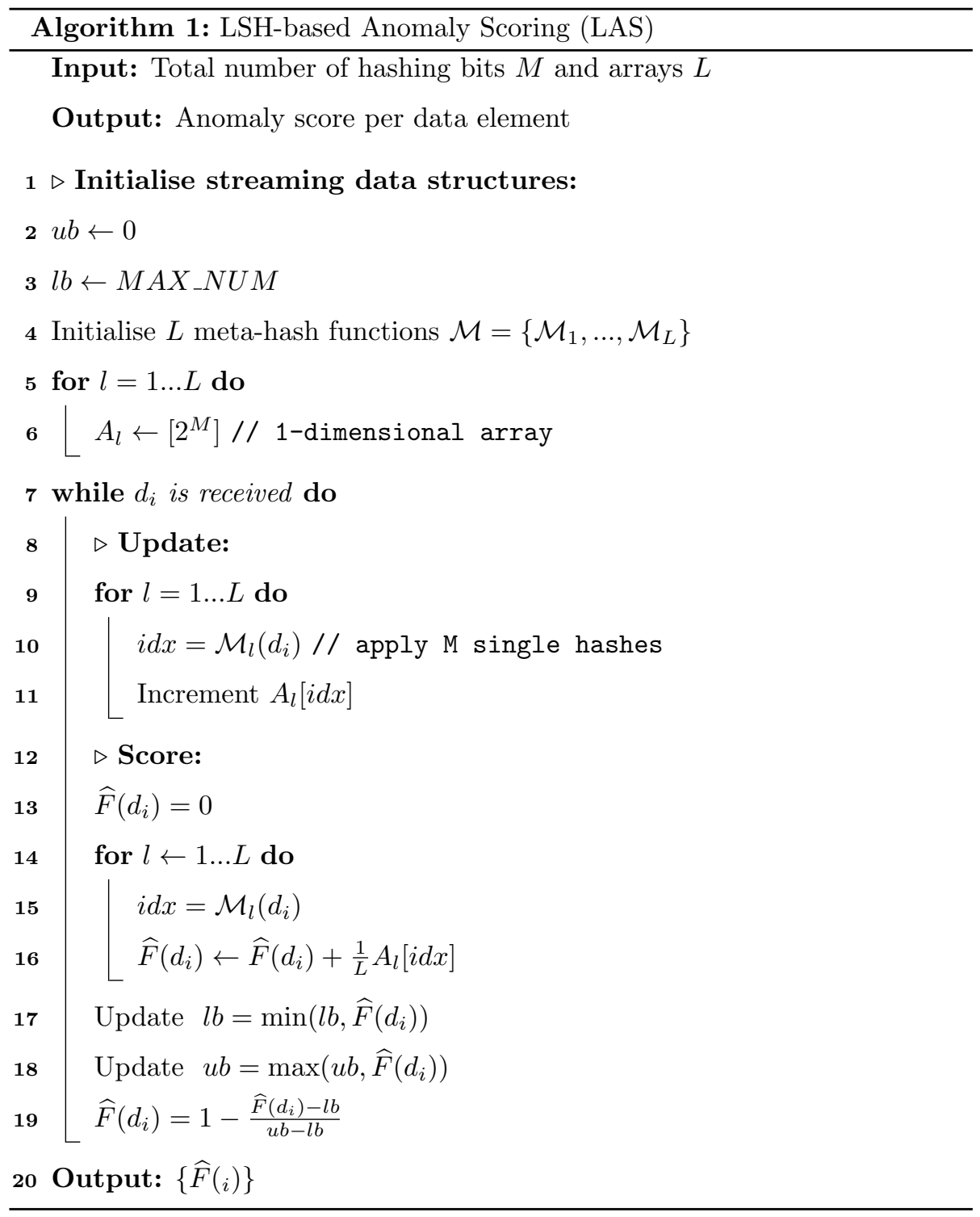

\subsection{Anomaly scoring process}

A streaming process of scoring anomaly signals in streaming data is described in Alg. 1. The algorithm starts with the initialisation of the data structure and the necessary variables (lines 2-6). During the processing of streaming data, the algorithm operates as a two-stage process as follows: 
- Update: Given any observed element, it increments the count of all the corresponding counters (line 8). Thus, every bit keeps the total number of collisions occurring at a particular index (Figure 4). The complexity of updating when each data item arrives is computed by $M \times L$ computations of single hashing accompanied by $L$ increments.

- Score: Given the updated sketch structure, we want to compute the score of the current data element (line 16). The overall cost for scoring is $M \times L$ computations accompanied by $L$ lookups, followed by a simple average calculation.

In general, the complexity of Alg. 1 is $\mathcal{O}(2(M L+L))$ for each incoming data element. Although we maintain each sketch for each entity or relation type for computing the anomaly score, the same size is applied for all sketches. Thus,

the algorithm is estimated based on the same collision probability (as revealed in Alg. 1) and yields a unified anomaly score for data elements from different modalities (R1). In the end, the anomaly score is normalized into $[0,1]$ (line 19) to reflect a likelihood (the higher the value, the higher the confidence that the data is an anomaly).

\section{High-order anomaly scoring}

In this section, we first present the structure of a rumour (Section 6.1) before shifting to high-order anomaly scoring (Section 6.2). We then show how †o extract a high-order rumour structure with a maximum anomaly score $\mathrm{Sec}$ tion 6.3.

\subsection{Rumour structure}

As demonstrated in Section 3.2, rumours in social networks appear as graph patterns (i.e., subgraphs) rather than single elements. For a generic representation, we model a rumour structure as a connected subgraph that contains anomalous elements. As a result, the scoring function of a rumour structure should respect the following aspects: 
- Direct connectivity. The ultimate goal of rumour detection is to report rumourous entities. However, relations should be incorporated into the score model, as they provide useful auxiliary information to eliminate false positives.

lousness of $S$. We use the idea of scan statistics [36, which computes the statistical significance of a set of probability values:

$$
\widehat{H}(S)=\max \bigcup_{\alpha_{m i n} \leq \alpha<1}\left\{\phi\left(\alpha,\left|D_{\alpha}(S)\right|,|D(S)|\right)\right\}
$$

where $\alpha_{\min } \in[0,1)$ is a pre-defined confidence threshold. For example, if we want to extract a rumour with $95 \%$ confidence, we set $\alpha_{\min }=0.95 . D(S)=$ $\{V(S) \cup E(S)\}$ is the set of elements in $S$, and $D_{\alpha}(S)=\left\{V_{\alpha}(S) \cup E_{\alpha}(S)\right\}$ is the set of elements $\{x \in D(S): \widehat{F}(x) \geq \alpha\}$ with $\alpha$-confident first-order anomaly scores. The reason for maximising $\alpha_{\min } \leq \alpha<1$ is that we want to find a sweet spot in the trade-off between direct connectivity and indirect connectivity.

The nature of rumour spreading is dynamic. Insignificant elements can become significant and vice versa. To maximise the completeness of detection, 'insignificant' elements $\left(D(S) \backslash D_{\alpha}(S)\right)$ connected with 'significant' elements $\left(D_{\alpha}(S)\right)$ should be included to reach the further end of rumour propagation. To 
reflect this, we model the function $\phi($.$) based on Kullback-Leibler divergence,$ which is non-parametric (R2). More precisely, the trade-off between $|D(S)|$ and $\left|D_{\alpha}(S)\right|$ can be modelled as [37:

$$
\phi\left(\alpha,\left|D_{\alpha}(S)\right|,|D(S)|\right)=|D(S)| \times K L\left(\frac{\left|D_{\alpha}(S)\right|}{|D(S)|}, \alpha\right)
$$

where $K L(x, y)=x \log \left(\frac{x}{y}\right)+(1-x) \log \left(\frac{1-x}{1-y}\right)$. As a result, our goal is to extract large subgraphs (via $|D(S)|$ ) with high confidence levels of anomaly (via $\left.\left|D_{\alpha}(S)\right| /|D(S)|\right)$.

Proposition 1 Computing $\widehat{H}(S)$ can be done by discretising first-order anomaly scores:

$$
\widehat{H}(S)=\max \underset{\alpha \in\{\widehat{F}(v): v \in S\} \cup\left\{\alpha_{\min }\right\}}{\bigcup}\left\{\phi\left(\alpha,\left|D_{\alpha}(S)\right|,|D(S)|\right)\right\}
$$

Proof 1 (Sketch) This property is inferred from the fact that $D_{\alpha}(S)$ is constructed by thresholding first-order anomaly scores in $D(S)$. Therefore, iterating $\alpha$ through distinct values of anomaly scores is sufficient, since the threshold in between two consecutive anomaly scores does not change $D_{\alpha}(S)$.

Example 3 A subgraph $S$ has entities $V(S)=\left\{v_{1}, v_{2}\right\}$ with $\widehat{F}\left(v_{1}\right)=0.98$, $\widehat{F}\left(v_{2}\right)=0.96$ and relations $\mathcal{E}(\mathcal{S})=\left\{e_{12}\right\}$ with $\widehat{F}\left(e_{12}\right)=0.99$. We choose $\alpha_{\text {min }}=0.95$. We have $|V(S)|=3$. With $\alpha=0.95$, we have $\left|V_{0.95}(S)\right|=3$ and $\phi(0.95,3,3)=3 \times(1 \log (1 / 0.95)+0 \log (0 / 0.05))=0.07$. With $\alpha=0.99$, we have $\phi(0.99,1,3)=3.18$. With $\alpha=0.97, \phi(0.97,2,3)=0.72$. With 95\% confidence, we can say that the anomaly score of $S$ is $\widehat{H}(S)=\max \{0.07,3.18,0.72\}=3.18$.

\subsection{Extracting high-order rumour structure}

Given a root subgraph (which can be a single element), Alg. 2 attempts to expand this subgraph to its neighbour. The expansion continues until it cannot further improve the high-order anomaly score. On the one hand, this neighbouring expansion acts as a cross-check between connected anomaly signals to construct a rumour structure. On the other hand, the returned subgraph is guaranteed to be maximal. 


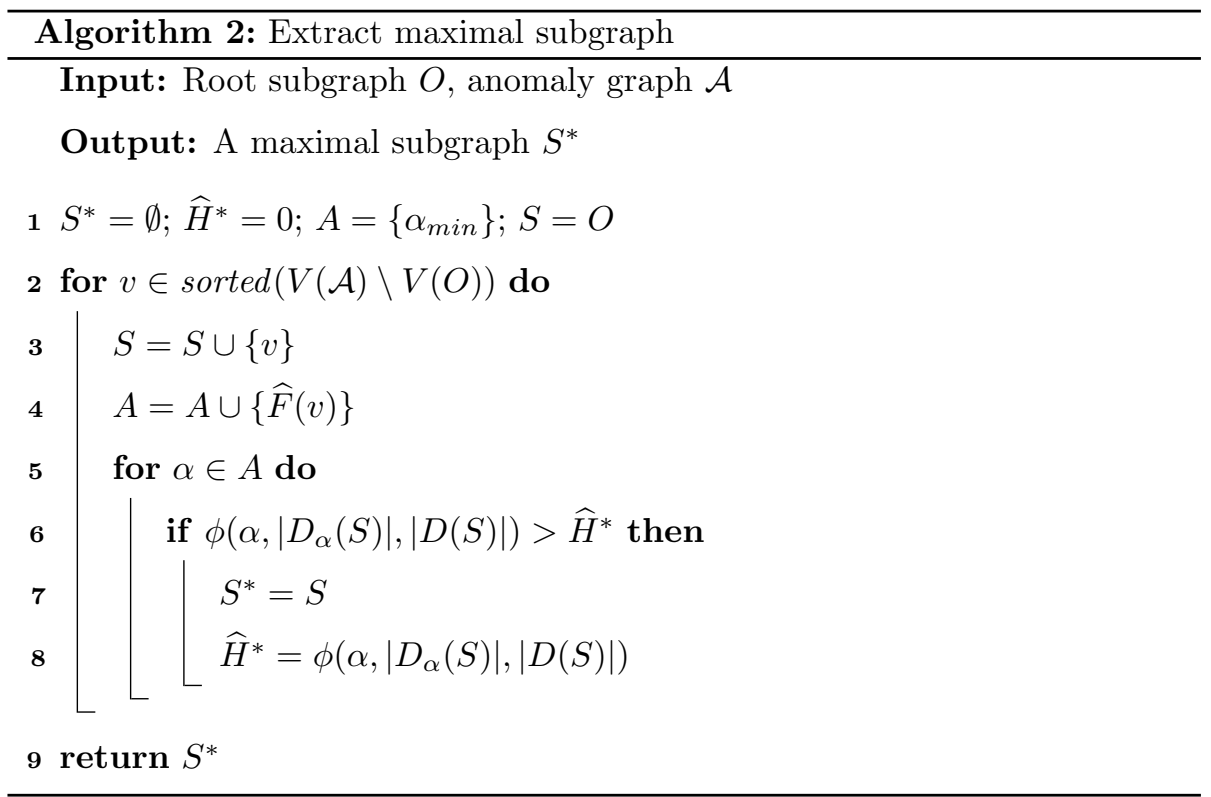

More precisely, Alg. 2 takes as input a root subgraph $O$ and a social graph with first-order anomaly scores $\mathcal{A}$. The returned subgraph is stored in variable $S$, which is initialised by $O$ (line 1). We go through the remaining entities in the increasing order of their first-anomaly scores as candidates to add into $S$ (line 3). On the one hand, we want the boundary of the subgraph as entities, since returning a relation without including one of its entities does not really make sense. On the other hand, adding entities by the increasing order of anomaly scores avoids local optima. At the end, the algorithm selects the maximal subgraph by maximising the high-order anomaly score $\widehat{H}(S)$ line 6 . However, there are two limitations of this algorithm. First, the returned subgraph is not guaranteed to be fully connected. Second, the number of remaining entities $V(\mathcal{A}) \backslash V(O)$ might be too large, making the iteration as well as the sorting slow. In the next section, we show that these limitations can be mitigated in an 'explore vs exploit' process. 


\section{End-to-end process}

Combining first-order (Section 5) and high-order (Section 6) anomaly scoring processes, we put them all together in a comprehensive end-to-end algorithm for rumour structure detection. Formally, we aim to detect a top list of maximal subgraphs that are solutions to the following optimisation:

$$
\underset{S \in \mathbb{S}(\mathcal{A})}{\arg \max } \widehat{H}(S)
$$

where $\mathbb{S}(\mathcal{A})$ is the set of all connected subgraphs of $\mathcal{A}$.

Proposition 2 Solving Eq. 8 is NP-hard.

Proof 2 (Sketch) We design a weight function $w: D(S) \rightarrow\{0,1\}$ with two cases: if $\widehat{F}(d) \geq \alpha$ then $w(d)=1$; otherwise $w(d)=0$. Since $\phi($.$) is mono-$ tonic with $\sum_{d \in S} w(v)$ via $\left|D_{\alpha}().\right|, E q .8$ is equivalent to the Maximal Weighted Common Subgraph problem, which is NP-hard [9, 38].

In our streaming setting, the solution to the above problem changes dynamically. It is wiser to maintain a list of top solutions instead. This point of view also relaxes the computational expense of the original problem, as a worse solution can become a better solution later on.

Alg. 3 implements this idea in an end-to-end manner. It runs on top of 40 an anomaly graph $\mathcal{A}$, a number of hops $Z$ (we take $\log |V|$ by default), and a coverage threshold $K($ default $=5)$. It returns a sorted list of the maximal subgraphs. First, it loops through entity types to make sure we capture rumours originated with different types of entities (line 4). Again, it does not make sense to select relations as spreading seeds. Second, we look through the top- $K$ entities with the highest first-order anomaly scores (line 5). This avoids missing rumours originated from different parts of the social graph. Then, it expands in $Z$-hop the seed entities to maximal subgraphs via Alg. 2 (line 9). The trick here is that we expand the subgraphs incrementally with the next-hop neighbourhood (line 8), which overcomes the connectivity and computation limitations of Alg. 2

so Specifically, we only need to sort the entities once (line 2). Finally, we include these maximal subgraphs as the output (line 12). 


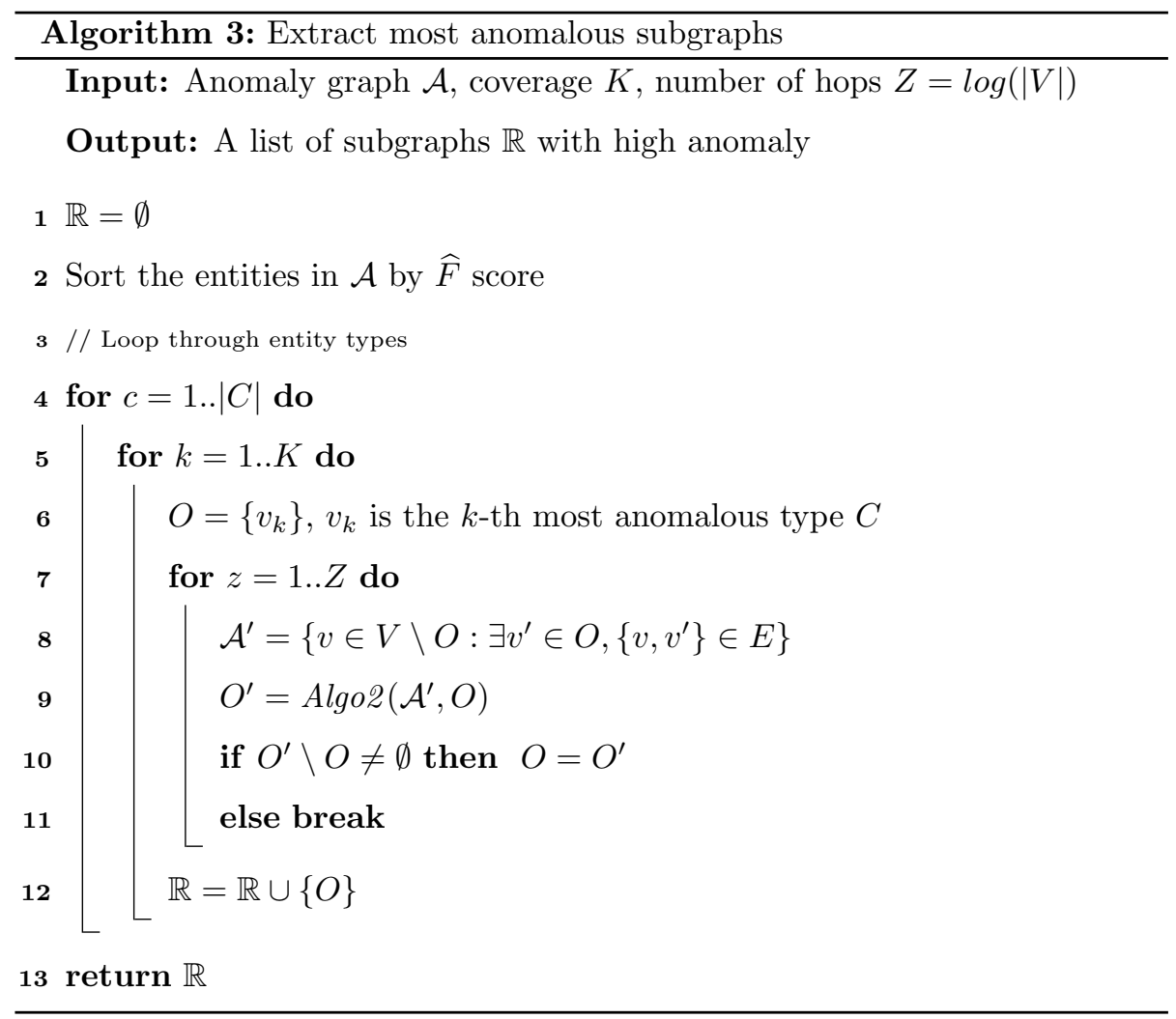

\section{Adaptive process}

We further explore how to adapt the process with concept drift Section 8.1 and input rate Section 8.2) to enhance the robustness of the system.

\subsection{Adaptive to concept drifts}

First-order concept drifts. Recall that anomaly scoring is based on comparing the abnormal values of rumour data against non-rumour data. However, in a streaming setting, the distribution of data may show concept drift, i.e., be non-stationary [39]. In other words, abnormal values in the past can become normal and vice versa. The challenge is that we cannot store all historical data to identify concept drifts in a streaming setting. 
To overcome this challenge, we propose a dual data sketch to capture the change of anomalies over a long period and a short period simultaneously. The reason behind this is that newly emerging rumours may have different data distributions than old rumours and thus their anomaly distribution is different. This characteristic of rumour has been well-studied in many previous works [40, 2. More precisely, we maintain two versions of the sketch for each entity or relation type. Let $\widehat{F}_{a}$ be the anomaly score computed throughout the beginning of the data stream. At the same time, $\widehat{F}_{c}$ captures the anomaly score for only the data elements within the current time window $W$. It is worth noting that the $\widehat{F}_{c}$ shall be reset every time we transition to the next time window. At anytime, we can estimate $\widehat{F}_{a}$ and $\widehat{F}_{c}$ using Alg. 1. We combine both scores with the bellow aggregation to be the final score for our anomaly scoring model:

$$
\widehat{F}\left(v_{i}\right)=\beta \cdot \widehat{F}_{c}\left(v_{i}\right)+(1-\beta) \cdot \widehat{F}_{a}\left(v_{i}\right)
$$

where $\beta \in(0,1)$. The final score gives us the chance to focus on emerging topics by putting a higher $\beta$-weight trade-off between long- and short-term indicators (R3).

High-order concept drifts. In social networks, the propagation structure of rumours may change over time, which affects the output (subgraph) of highorder anomaly scoring. To handle such high-order concept drift, we maintain a list of potential rumours and continuously update them upon the arrival of new data. More precisely, when the graph has a new element, we check which maximal subgraphs it is connected to. This is efficiently implemented by associating each element of a maximal subgraph that is output by Alg. 3 to the identifier of the seed entity. As a result, we can associate a new node with existing rumours via its neighbours. Since the new element can increase or decrease the high-order anomaly score, the new induced subgraphs can be rumours or not.

If the anomaly score of the new element is higher than any scores in an associated rumour, the induced subgraph is definitely a rumour. This is because the anomaly score of the whole subgraph will increase. Otherwise, when the new 
the induced subgraph might not be a rumour anymore since its anomaly score

decreases. As a result, we need to re-run the seed expansion step of Alg. 3 (lines 6-11) to detect rumour. This is possible due to the undirected property of our algorithm, such that the expansion can be done in any direction. Further, the expansion step consolidates the level of confidence to conclude whether a structure is a rumour or not. In other words, only when the expansion takes place, a structure could be outputted as a rumour or kept in the queue as a potential rumour.

\subsection{Adaptive to different input rates} timeliness of rumour detection [41. In fact, the first-order and the high-order strategies for anomaly scoring induce a trade-off. Using only the former may lead to a disconnected set of rumourous elements. However, an excessively high-order approach may increase the number of non-rumourous elements. To

mitigate this trade-off, we propose a dynamic alternating procedure based on two factors:

- Ratio of non-rumourous elements. If the number of non-rumourous elements becomes large, the first-order strategy is preferred. When a rumour is emerging (high data rate), rumourous elements often appear connected to each other. This is because rumours propagate fast and widely [2] As such, the first-order strategy is favoured in such cases to quickly detect rumours.

Formally, the ratio of non-rumourous elements in a set of output subgraphs $\mathcal{R}$ is:

$$
\delta(\mathcal{R})=\sum_{R \in \mathcal{R}} \frac{|D(R)|-\left|D_{\alpha}(R)\right|}{|D(R)|}
$$

- Clustering anti-coefficient. To measure the connectivity of rumourous elements, we use the global clustering anti-coefficient 42. Unlike the local clustering anti-coefficient 43, which indicates the connectivity of a single 
node, the global clustering anti-coefficient reveals the connectivity of the whole graph structure. This global measure is suitable for our problem due to the characteristics of rumour propagation (e.i., a rumourous element could be spread together with non-rumoumous elements from any direction rather than from a specific element). When the connectivity is too small, the high-order strategy is preferred to preserve the propagation structure of rumours. When a rumour is not widespread yet (low data rate), the high-order strategy is favoured since we have time to run more complex rumour detection.

Formally, the clustering anti-coefficient [42] is defined as:

$$
\epsilon(\mathcal{R})=\sum_{R \in \mathcal{R}}\left(1-\frac{1}{|R|} \sum_{v \in R} \frac{\# \text { triangles on } \mathrm{v}}{\# \text { triples on } \mathrm{v}}\right)
$$

The choice between the two strategies is then defined with a likelihood score:

$$
z=1-e^{-(\epsilon(1-h)+\delta h)}
$$
recompute the likelihood $z$ line 16 to shift the trade-off with new information. 


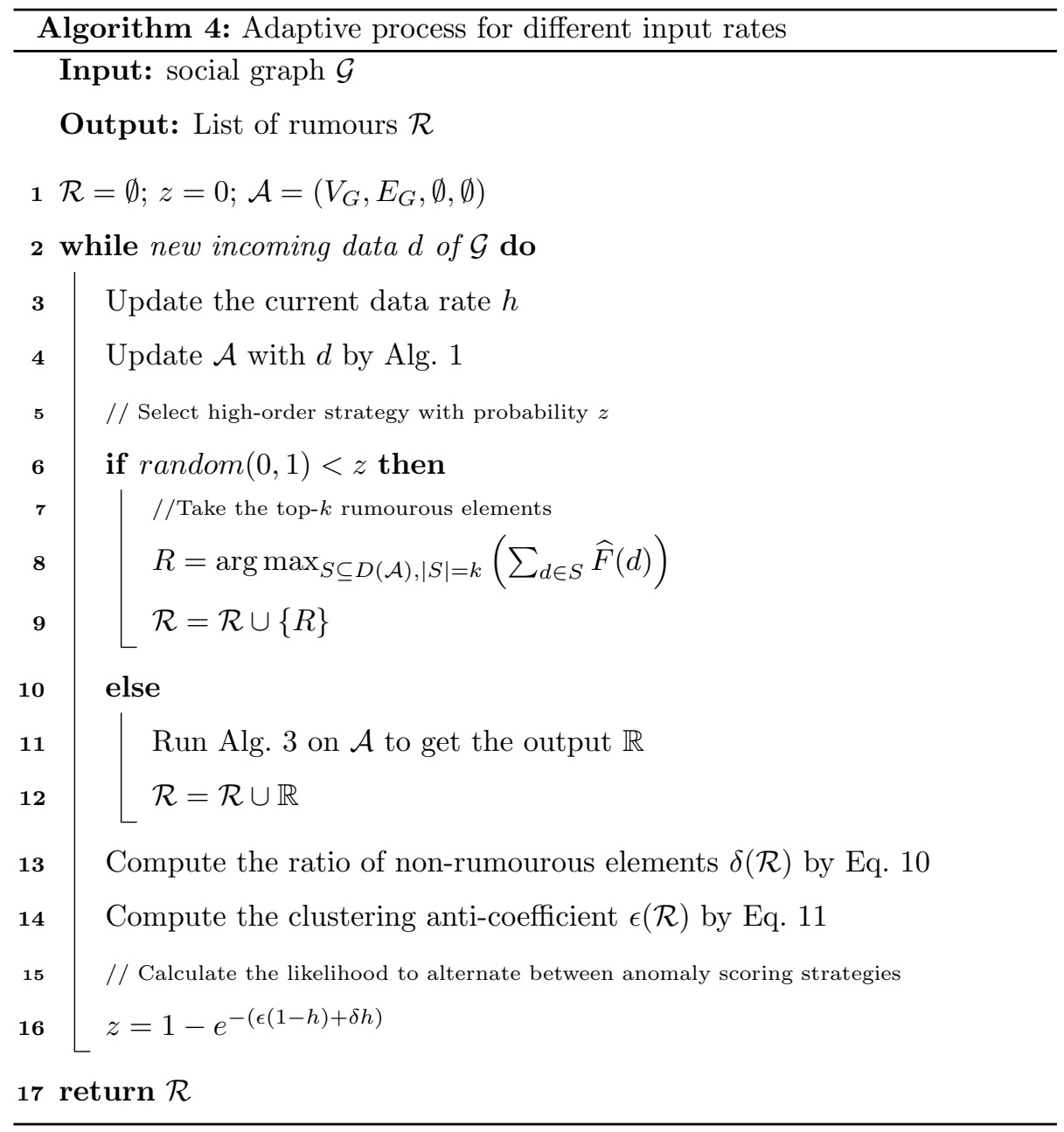

\subsection{Complexity analysis}

Since we model the social platform as a multi-modal social graph, we rely on graph parameters to evaluate the complexity of the proposed solution. Without any loss of generality, we focus on several factors: $N=|V|+|E|$ as the number of elements on the social graph; $D_{\max }$ as the graph maximal degree; the number of hashing bits $M$ and arrays $L$ from Alg. 1 the number of entity types $C$, the coverage $K$, and the number of hops $Z$ in Alg. 3 .

575 Time complexity. There are two main steps to analysis: 
- First-order anomaly scoring (Alg. 1): Recall that the first-order anomaly scoring function consists of two main actions (update and score), each of which takes $M L$ computations of single hashing followed by $L$ increments. In other word, it takes $\mathcal{O}(2 L(M+1))$ to evaluate an incoming data element. So, the computations for $N$ elements are $\mathcal{O}(2 N L(M+1))$, and this amount is doubled to $\mathcal{O}(4 N L(M+1))$ by adopting the dual-sketch.

- High-order anomaly scoring (Alg. 3): For each rumourous candidate, the algorithm tries to expand to obtain the maximal anomalous subgraph. Each expansion step could bring $D_{\max }$ relations associated with another $D_{\max }$ entities into consideration. In this way, the computations for $Z$ hops expansion are $\mathcal{O}\left(2 Z D_{\max }\right)$. The algorithm covers $K$ candidates for each entity type $C$; thus the total computations are $\mathcal{O}\left(2 C K Z D_{\max }\right)$.

Therefore, the overall time complexity is $\mathcal{O}\left(4 N L(M+1)+2 C K Z D_{\max }\right)$. It is worth nothing that other parameters could be dominated the number of elements $N$ in the social graph; thus, the overall time complexity is simply rewritten as $\mathcal{O}(N)$.

Space complexity. There are two main steps to analysis:

- First-order anomaly scoring Alg. 1): The algorithm only maintains a constant memory of $M L$ for each sketch. In such a manner, the space complexity for the dual-sketch is $\mathcal{O}(2 M L)$.

- High-order anomaly scoring (Alg. 3): We need to maintain the maximal elements of $\mathcal{O}\left(2 C K Z D_{\max }\right)$ in memory for high-order scoring.

Thus, the framework needs a constant space of $\mathcal{O}\left(2\left(M L+C K Z D_{\max }\right)\right)$.

\section{Empirical evaluation}

We conduct experiments on top of our rumour benchmarking datasets. We first discuss the experimental setting (Section 9.1) and then assess different aspects of our approach: 
- The end-to-end performance of our framework Section 9.2

- The evaluations on first-order rumour detection (Section 9.3)

- The evaluations on high-order rumour detection (Section 9.4)

- The performance of the ablation tests Section 9.5

- The robustness to concept drifts Section 9.6)

- The performance of the adaptive process framework (Section 9.7)

- The analysis of hyperparameter sensitivity (Section 9.8)

- The discussion and practical guidelines (Section 9.9)

\subsection{Experimental setting}

Datasets. We use our published benchmark of rumour data from Twitter and Snope [9]. It contains 3 million users, 4 million tweets, 305115 linked articles, and 28893 hashtags, including around 1022 rumours. The data spans several domains, each of which is a separate dataset:

- Politics: contains rumours associated with political issues.

- Crime: contains crime-related rumours.

- SciTech: contains rumours that are false technological inventions or scientific myths.

${ }_{620}$ Metrics. We employ a fine-grained level of metrics in our evaluation:

- The detection coefficient [44: A balanced metric between the accuracy and coverage of rumour detection in the context of graph data. We denote the output of our anomaly scoring problem as $\mathcal{R}$, while the ground-truth is $\mathcal{R}^{*}$. Then, the coefficient is the following ratio: $\frac{\left|\mathcal{R}^{*} \cap \mathcal{R}\right|}{\left|\mathcal{R}^{*} \cup \mathcal{R}\right|}$.

- The run-time: The total computational time of running the given detection algorithm on a social graph.

- The lag time to detection: The delay between the detection of a rumour and its genesis.

Baselines. Most evaluations of rumour detection [4 focus on classification metrics (all elements in the social graph need to be classified as a rumour or not). However, a rumour may not contain a single element. Additionally, some 
elements can become rumourous after being affected by a rumour over time. In our setting, the output is a list of subgraphs, each of which reflects the propagation structure of a rumour. Against this background, we adapt several baseline methods based on their respective papers.

- GraphScan: An anomaly-based rumour detection technique [9] for whole graph data. It scans for subgraphs with high inconsistency signals. We use its early detection version, which maintains a list of candidate subgraphs and marks them when they become true rumours.

- Pattern: A subgraph-based anomaly detection technique that matches streaming data against rumour patterns [11, 12. However, a disadvantage of this technique is that the patterns need to be defined beforehand and there is a limited number of them.

- o-Classifiers: An ensemble of the decision-tree classifier [45], SVM-based classifier [46, rank-based classifier [10, and sequential models [7. We use their online versions to be compatible with streaming data.

Hyperparameter setting. If not stated otherwise, the hyperparameters are chosen as follows: $M=15, L=50$ (the number of projections), $\beta=0.8$ (the weight for the trade-off between the short- and long-term sketch), the time window $W$ for the short-term sketch is set to 12 hours, $K=15$ (the coverage of the most anomalous element). On the other hand, we also study the analysis of hyperparameter sensitivity in Section 9.8 .

Environment. We use an Intel Core i7 system (2.8 GHz, 16GB RAM) to conduct experiments. If not specified otherwise, all experiments are averaged over 10 runs and all datasets to avoid randomness.

\subsection{End-to-end process}

\subsubsection{Efficiency}

We evaluate the running time of our approach versus the baselines with different graph sizes. To this end, we generate random graphs from the original graph with the Erdos-Renyi model given a pre-defined number of entities. 


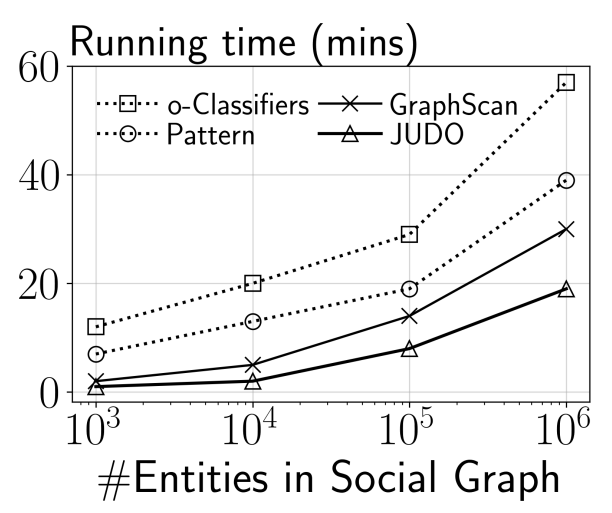

Figure 5: Efficiency vs effectiveness.

Figure 5 depicts the results averaged over all datasets, where the $\mathrm{X}$-axis is the number of entities and the $\mathrm{Y}$-axis is the running time. In general, we see that our approach achieves a much higher efficiency $($ JUDO $=19 \mathrm{~min})$ compared to the best baseline (GraphScan $=30 \mathrm{~min}$ ). This improvement originates from the superiority of the online indexing mechanism of anomaly signals (as proposed in Section 5.3. While the Pattern gives a moderate running time, it yields a much lower detection coefficient (as reported in the section below). o-Classifiers is the least efficient over all other baselines.

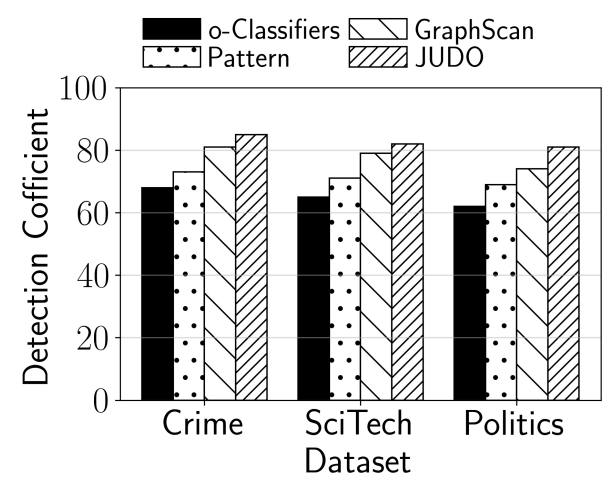

Figure 6: Effectiveness of rumour detection techniques. 


\subsubsection{Effectiveness} over each dataset. The key finding is that our approach outperforms the baselines in detecting rumours. For instance, in the Politics dataset, JUDO reaches a detection coefficient of 0.81 while the best runner-up (GraphScan) only has a coefficient of 0.78 . The same trend is observed in the remaining datasets (Crime

\subsubsection{Timeliness}

Table 3 depicts the timeliness of rumour detection of different methods. The analysis is performed within $12 \mathrm{~h}$ after the genesis of rumours. We measure the average lag time to detection for all rumours. Overall, we see that our approach detects at least $8 \%$ more rumours and detects them at least six times faster on average within the same time frame.

Table 3: Timeliness of rumour detection (within 12h).

\begin{tabular}{lcc}
\hline Baseline & Rumours detected & Average lag time (hour[s]) \\
\hline o-Classifiers & $65 \%$ & +2.93 \\
Pattern & $70 \%$ & +1.09 \\
GraphScan & $76 \%$ & +0.67 \\
JUDO & $\mathbf{8 2 \%}$ & $\mathbf{+ 0 . 1 2}$ \\
\hline
\end{tabular}

\subsection{Evaluations on first-order anomaly scoring}

\subsubsection{Correctness of anomaly scores}

In this experiment, we assess the efficacy of first-order anomaly scoring. As we use the first-order scoring to validate the rumour-related tweets, the scoring should reflect the ground truth (the lower the score, the more rumourous an entity). Figure 7 shows a histogram of anomaly scores between non-rumour and rumour-related elements. The result is averaged over all datasets. The X-axis represents the discretised value of the first-order normalised anomaly score, and 
the score $\geq 0.95$ is high. We see that most of the correct non-rumour related tweets have occurred with an anomaly score of $\leq 0.9$.

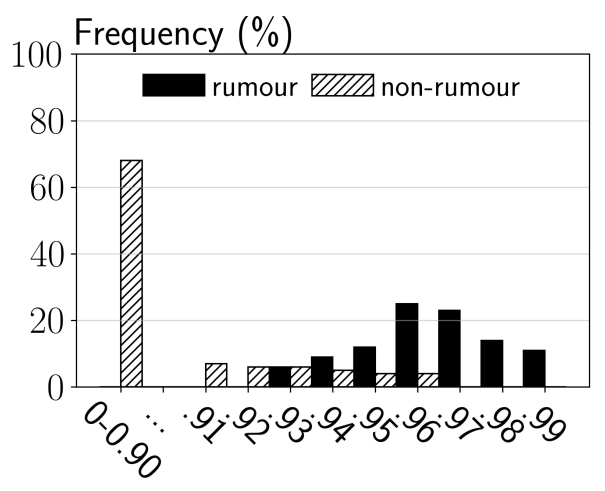

1st-order score

Figure 7: Correctness of first-order anomaly scoring.

9.4. Evaluations on high-order anomaly scoring

9.4.1. Correctness of anomaly scores

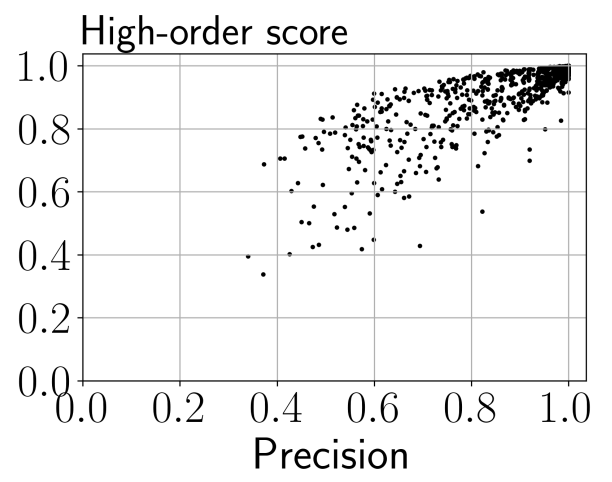

Figure 8: Correctness of high-order anomaly scoring.

We verify our rationale that the high-order anomaly score is correlated with the precision of rumour detection. In this experiment, the high-order detection was executed 100 times per dataset (300 runs in total), and Figure 8 describes the results. The $\mathrm{X}$-axis represents the precision of the current trial, while the 
Y-axis depicts the normalised high-order score value of all detected subgraphs. There is a strong positive correlation between both values. Hence, the highorder score is indeed a reliable indicator for the detection of rumour structure.

\subsubsection{Explore vs exploit effect}

Figure 9 compares the correctness of the first-order and high-order scoring functions. In the first-order function, we consider elements with the score $\geq$ 0.95 as anomalies. Although the strategy is straightforward and yields a high precision, the recall is low as evaluating a rumour using such isolated elements is unreliable. Hence, the first-order technique is an exploitation. In contrast, the high-order function is an exploration technique because it tries to capture the propagation structure of the rumour. By doing so, the high-order could yield higher per-entity false alarms; however, it is necessary to incorporate some nonrumourous elements because, e.g., an innocent user could accidentally spread a rumour.

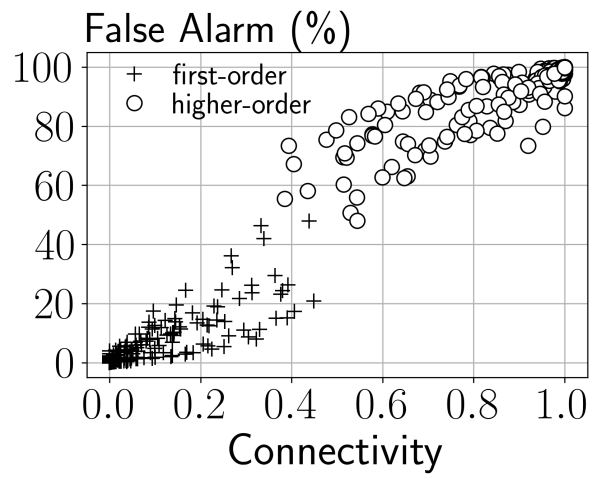

Figure 9: Correctness of first-order and high-order anomaly scoring.

\subsection{Ablation tests}

\subsubsection{Multi-level vs. single-level approaches}

In this experiment, we conduct the ablation test with six different settings, which are categorized into two groups. By doing so, we want to verify the importance of our multi-level approach compared to the feasible alternative 
(e.g., single-level approach). To this end, we measure the running time and detection coefficient of each setting in each group over the whole social graph, and the results are depicted in Table 4.

Table 4: Importance of each model component.

\begin{tabular}{|c|c|c|c|}
\hline & Setting & Running time (mins) & Detection coeff. \\
\hline \multirow{3}{*}{ 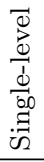 } & First-order (p-value) & 25 & 0.61 \\
\hline & First-order (Midas) & 8 & 0.41 \\
\hline & First-order (LSH) & 9 & 0.66 \\
\hline \multirow{3}{*}{ 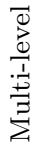 } & First-order (p-value) + high-order & 39 & 0.81 \\
\hline & First-order (Midas) + high-order & 21 & 0.58 \\
\hline & First-order $(\mathrm{LSH})+$ high-order & 22 & 0.83 \\
\hline
\end{tabular}

Overall, the results significantly improve (up to 27\%) when multi-level approaches are adopted for rumour detection. The superiority stems from the cross-checking and false-positive filtering of the high-order component. As the multi-level techniques are built on top of the single-level approaches, they take extra time to execute; however, it is a reasonable trade-off to achieve better overall performance.

We also assess the efficiency of our LSH-based anomaly indexing in multilevel settings. Overall, our approach results in the best trade-off between the performance and running time (0.83 for the detection coefficient in $22 \mathrm{~min}$ ). It can be seen that the p-value and high-order approach yields a reasonable performance (0.81), with a running time of 39 min. The setting with Midas produces an unsatisfactory performance, as our high-order approach could not help much in the case when the first-order detection is at a moderate level.

\subsubsection{Effect of first-order scoring function}

We further validate the influence of our LSH-based indexing component by replacing it with the feasible alternatives. More precisely, two representative baselines are adopted: p-value (i.e., an anomaly detector that exploits entitylevel statistical significance [9]) and Midas (i.e., anomaly detector Midas [30]). 
The comparison result is also depicted in Table 4. While the p-value detector gives a comparative coefficient to our LSH-based indexing structure, it takes a much longer time to complete (up to $\mathrm{x} 3$ times longer). Midas is indeed a little bit faster than ours; however, the performance drops significantly. The reason for this is that Midas is designed based on the idea of the counting sketch [47, which is only able to handle integer features. To use Midas for this comparison, some features with real values such as linguistic style and popularity are rounded before fitting the model. The information loss caused by the pre-processing step hinders the detecting power of Midas, which leads to the degradation of its overall performance.

\subsubsection{Effect of high-order scoring function}

Here, we study the importance of the high-order scoring function by comparing its performance to the feasible alternatives. The details of the alternatives are as follows:

- High-order 1: We consider the subgraph $S$ as a set of elements rather than a high-order struture of rumour. The alternative score $H^{\prime}(S)$ is the mean score of all elements.

- High-order 2: The scoring function is modified by replacing the tradeoff [37] between $|D(S)|$ and $\left|D_{\alpha}(S)\right|$ by the ratio $r=\left|D_{\alpha}(S)\right| /|D(S)|$. This new scoring function becomes parametric; thus a threshold of $r$ is applied to identify a rumour structure.

Table 5 shows the results. Overall, the high-order scoring function has the best detection coefficient. The reason for this is that it successfully discovers the best trade-off between the direct and indirect connectivity of the rumour structure. The alternatives run a bit faster due to their simpler calculations. High-order 1 performs the worst. When considering the subgraph $S$ as a set of isolated elements, it could not capture the propagation characteristics, which is crucial for rumour detection 40. High-order 2 favours direct connectivity 
between significant elements, thus leading to more false-positives than the tradeoff strategy.

Table 5: Effect of high-order scoring function.

\begin{tabular}{lcc}
\hline Setting & Running time (mins) & Detection coeff. \\
\hline High-order 1 & 11 & 0.71 \\
High-order 2 & 11 & 0.74 \\
High-order & 13 & 0.83 \\
\hline
\end{tabular}

\subsection{Effects of concept drifts} a miss rate lower than $20 \%$ when the data arrival reaches $40 \%$, and the system remains stable from that point on. In contrast, the no adaptivity version needs more data (i.e., $80 \%$ ) to reach a similar level of stability. 


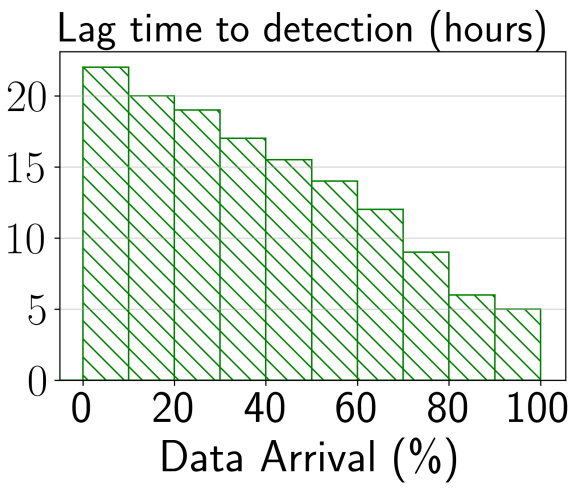

(a) With concept drift adaptivity

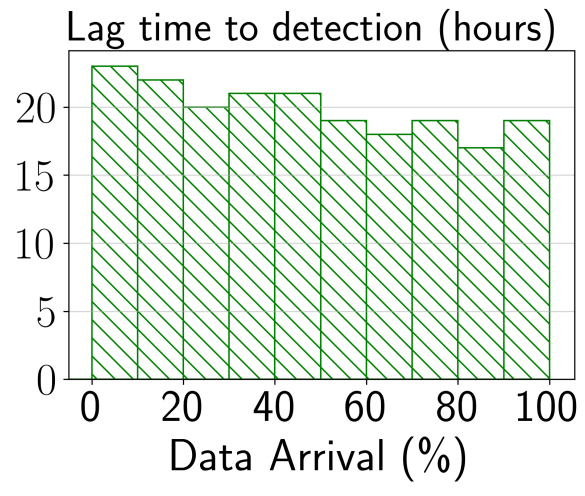

(b) W/o concept drift adaptivity

Figure 10: Concept drift effect on first-order detection.

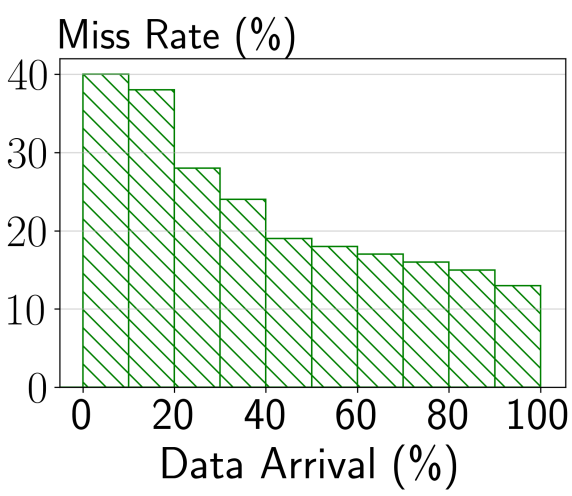

(a) With concept drift adaptivity

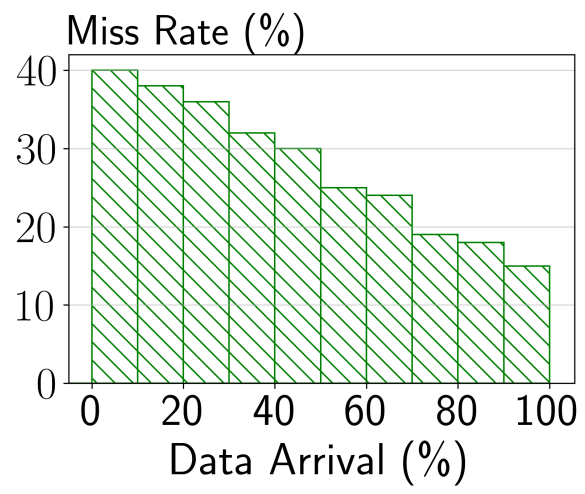

(b) W/o concept drift adaptivity

Figure 11: Concept drift effect on high-order detection.

\subsection{Input rate adaptive process}

\subsubsection{Comparative analysis for input rate adaptive process}

In this section, we assess the performance of the adaptive process with another two feasible alternatives: adapting the input rate to the ratio of nonrumourous elements only, and adapting the input rate to the clustering anticoefficient only. Three settings are conducted with a high input rate. Table 6 presents the comparative results. Overall, the adaptive process achieves the best detection coefficient. This result confirms a clear advantage when applying 
the combination of the two strategies. The ratio of non-rumourous elements strategy yields a moderate performance. Without the cross-checking of the high-order detector, it might include rumour-like phenomena such as spam and hype [48. When only the clustering anti-coefficient is employed, the process is inefficient when it continuously checks for a non-rumourous subgraph which turns out to have a non-rumour structure.

Table 6: Effect of input rate.

\begin{tabular}{lc}
\hline Setting & Detection coeff. \\
\hline Ratio of non-rumourous elements & 0.78 \\
Clustering anti-coefficient & 0.76 \\
Input rate adaptive process & 0.82 \\
\hline
\end{tabular}

\subsubsection{Effect of input rate adaptive process}

Table 7 presents the sensitivity of our approach against different data distributions (normal and skewed) and input rates (low and high). We see that the first-order scoring is less sensitive to input rate, but it is sensitive to the data distribution (i.e., the detection coefficient decreases in skewed data). The highorder scoring in combination with first-order scoring (as in the normal process) gives a better performance compared to the first-order scoring only.

Another finding is that the detection coefficient slightly degrades when the input rate is high. This is because the high input rate forces the first-order strategy to be more favoured in most cases for timely detection (Section 8.2). As a result, the data items are often assessed individually rather than collectively, making the detection of rumours as subgraphs less accurate. We have also tested the case where the high-order strategy is adopted randomly; however, it yields a poor result due to the trivial approach. Finally, as expected, the adaptive process gives a good trade-off in all cases. 
Table 7: Detection coefficient against adversarial conditions.

\begin{tabular}{clccc}
\hline data dist. & input rate & first-order only & normal process & adaptive process \\
\hline \multirow{2}{*}{ normal } & low & 0.66 & 0.83 & $\mathbf{0 . 8 4}$ \\
& high & 0.65 & 0.79 & $\mathbf{0 . 8 2}$ \\
\hline \multirow{2}{*}{ skewed } & low & 0.63 & 0.78 & $\mathbf{0 . 8 0}$ \\
& high & 0.62 & 0.77 & $\mathbf{0 . 7 9}$ \\
\hline
\end{tabular}

\subsection{Hyperparameter sensitivity}

In this section, the important hyperparameters are evaluated based on the sensitivity of the results to these parameters.

Number of projections $(M)$. We experiment to validate the discriminative power [49] of the local scoring function Eq. 5). More precisely, for a social graph, we define abnormal elements as elements related to any rumour and normal elements as elements related to no rumour.

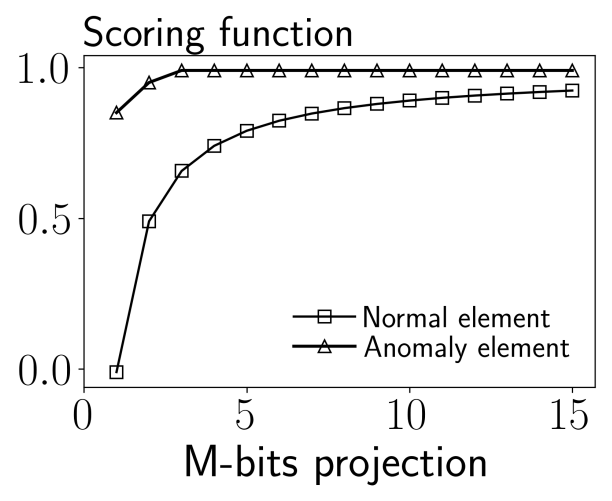

Figure 12: Discriminative power of first-order scoring function.

All elements are passed through the scoring function and the average scores are presented in Figure 12 with different numbers of projections. As we can see from the results, the score of the abnormal elements is quite near one when the number of projections increases. However, there is a trade-off. With a small number of projections, the distinction is large but the score of the abnormal entities may not reach a $95 \%$ confidence level. $M=15$ seems to be a stable 
choice since the difference is recognisable while achieving a high confidence level. We apply the fixed value $L=50$ for all experiments.

$\beta$-weight for dual sketch. Figure 13 studies the sensitivity of the $\beta$-weight trade-off between the short-term and long-term sketches. It can be seen that the detection coefficient gradually improves when increasing the $\beta$-weight. However, when $\beta$ is greater than 0.8 , it does not increase the performance. In general, $\beta=0.8$ provides the best trade-off and yields the best detection coefficient in all three datasets.

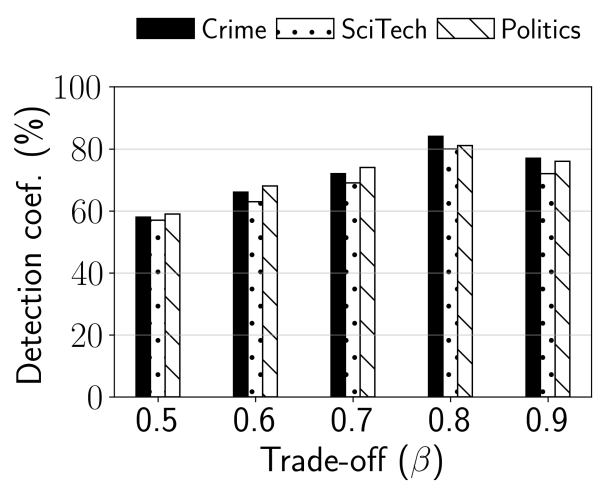

Figure 13: Sensitivity of $\beta$-weight for dual sketch.

${ }_{840}$ The coverage (K). In this experiment, we study the effect of the coverage (K) on the detection performance, and Figure 14 reveals the results. The analysis did not show significant differences in terms of the detection coefficient when varying the coverage $(K)$. However, $K=15$ brings in the finest result; it is used in other experiment settings throughout this section.

\subsection{Discussions}

Summary of the results. Our system modelled the social platforms using a social graph representation and monitored rumourous signals from both the element-level and subgraph-level. We empirically evaluate our approach for just-in-time rumour detection from different angles. For the single-level setting, the first-order detection efficiently scans the dataset within 9 min, achieving a 


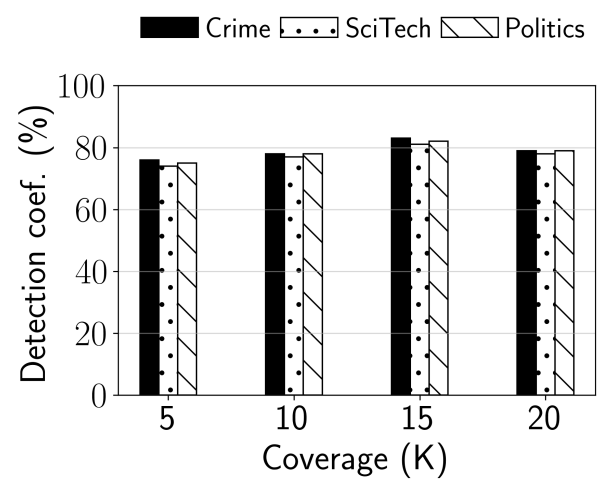

Figure 14: Sensitivity of the coverage (K).

detection coefficient of $66 \%$. For the end-to-end setting, with the complement of the high-order component, the detection coefficient increases to $82 \%$ and the average timeliness of detection is $0.12 \mathrm{~h}$. Also, the efficiency scales linearly with the graph size, while a constant amount of memory is required.

Practical guidelines. Although our approach has multiple levels and components, our framework is highly decoupled. Other researchers might easily enhance our study by adapting new methods for each component to achieve similar goals. Our framework is open to being adapted to new social platforms; however, one should use hyperparameter tuning to find the best configurations, which is a common strategy in data mining [50]. Others can also explore more types of concept drift and settings of the input rate relevant to their applications.

\section{Conclusion}

This paper proposes JUDO, a just-in-time approach for rumour detection in a streaming social platform, which is run on top of the continuous scoring of rumour-related signals. The contributions of this study can be revealed in terms of both methodological and practical viewpoints.

From the methodological perspective, we go beyond state-of-the-art approaches, which neglect the early aspect of detection. More precisely, we focus 
damage. To this end, we formulate the problem of anomaly scoring for just-intime rumour detection, where potential rumours are scored by their abnormal behaviours and detected when they emerge. Our solution to this problem includes two levels of anomaly scoring: first-order signals and high-order signals.

875 The former tracks anomaly signals at the individual level and incrementally computes an anomaly score for each element, while the latter finds connected subgraphs with maximal collective scores that are likely to become rumours. Our solution is compatible with streaming data and even adaptive to different streaming conditions such as concept drift and a fluctuating input rate. Extensive experiments on a wide range of real-world datasets show that our approach outperforms the baselines with detection at least six times earlier and $8 \%$ more accurate detection within the $12 \mathrm{~h}$ time frame.

From a practical perspective, our findings address a real and important problem in social media, where rumours are spread any time. Just-in-time rumour detection provides timely precautions to minimize the negative impact of rumour spreading. In practice, one could extend this study's results to a webbased observatory dashboard that helps media and government organizations to distinguish between legitimate and deceptive content. In addition, other researchers can seamlessly integrate our framework with the rumour resolution pipeline (i.e., rumour detection, rumour tracking, and veracity classification) [4] to encourage more investigations in the community of rumour research.

The limitations of our study could be encountered when a user is only interested in rumours concerning a specific topic and has a desire to sacrifice other topics to obtain the performance gain. In future works, we tend to enhance our framework to perform content-based rumour identification. More precisely, we shall employ a topic-based filtering and monitoring tool to explore and aggregate topic information prior to lifting data into the stream setting for rumour detection.

Acknowledgment. This work was supported by ARC Discovery Early Career 
Researcher Award (Grant No. DE200101465).

\section{References}

[1] A. Bondielli, F. Marcelloni, A survey on fake news and rumour detection techniques, Information Sciences 497 (2019) 38-55.

[2] S. Vosoughi, D. Roy, S. Aral, The spread of true and false news online, Science 359 (6380) (2018) 1146-1151.

[3] L. Yang, Z. Li, A. Giua, Containment of rumor spread in complex social networks, Information Sciences 506 (2020) 113-130.

[4] A. Zubiaga, A. Aker, K. Bontcheva, M. Liakata, R. Procter, Detection and resolution of rumours in social media: A survey, CSUR 51 (2) (2018) 32.

[5] K. Zhou, C. Shu, B. Li, J. H. Lau, Early rumour detection, in: Proceedings of the 2019 Conference of the North American Chapter of the Association for Computational Linguistics: Human Language Technologies, Volume 1 (Long and Short Papers), 2019, pp. 1614-1623.

[6] J. Ma, W. Gao, P. Mitra, S. Kwon, B. J. Jansen, K.-F. Wong, M. Cha, Detecting rumors from microblogs with recurrent neural networks.

[7] S. Han, Context-aware message-level rumour detection with weak supervision, Ph.D. thesis, University of Sheffield (2020).

[8] X. Zhou, R. Zafarani, Fake news: A survey of research, detection methods, and opportunities, arXiv preprint arXiv:1812.00315.

${ }_{920}^{9}$ [9] N. T. Tam, M. Weidlich, B. Zheng, H. Yin, N. Q. V. Hung, B. Stantic, From anomaly detection to rumour detection using data streams of social platforms, Proceedings of the VLDB Endowment 12 (9) (2019) 1016-1029.

[10] Z. Zhao, P. Resnick, Q. Mei, Enquiring minds: Early detection of rumors in social media from enquiry posts, in: Proceedings of the 24th international conference on world wide web, 2015, pp. 1395-1405. 
[11] S. Wang, T. Terano, Detecting rumor patterns in streaming social media, in: 2015 IEEE International Conference on Big Data (Big Data), 2015, pp. $2709-2715$.

[12] S. Wang, I. Moise, D. Helbing, T. Terano, Early signals of trending rumor event in streaming social media, in: 2017 IEEE 41st Annual Computer Software and Applications Conference (COMPSAC), Vol. 2, 2017, pp. 654659.

[13] L. Zhu, H. Zhao, H. Wang, Complex dynamic behavior of a rumor propagation model with spatial-temporal diffusion terms, Information Sciences 349 (2016) 119-136.

[14] A. I. E. Hosni, K. Li, S. Ahmad, Minimizing rumor influence in multiplex online social networks based on human individual and social behaviors, Information Sciences 512 (2020) 1458-1480.

[15] Y. Li, Z. Su, J. Yang, C. Gao, Exploiting similarities of user friendship networks across social networks for user identification, Information Sciences 506 (2020) 78-98.

[16] L. Zhu, B. Wang, Stability analysis of a sair rumor spreading model with control strategies in online social networks, Information Sciences 526 (2020) $1-19$.

[17] C. Shi, Y. Li, J. Zhang, Y. Sun, S. Y. Philip, A survey of heterogeneous information network analysis, IEEE Transactions on Knowledge and Data Engineering 29 (1) (2016) 17-37.

[18] M. R. Bouadjenek, H. Hacid, M. Bouzeghoub, Social networks and information retrieval, how are they converging? a survey, a taxonomy and an analysis of social information retrieval approaches and platforms, Information Systems 56 (2016) 1-18. 
[19] S. Sahu, A. Mhedhbi, S. Salihoglu, J. Lin, M. T. Özsu, The ubiquity of large graphs and surprising challenges of graph processing: extended survey, The VLDB Journal (2019) 1-24.

[20] J. Li, H. Jiang, X. Mei, C. Hu, G. Zhang, Dynamical analysis of rumor spreading model in multi-lingual environment and heterogeneous complex networks, Information Sciences 536 (2020) 391-408.

[21] M. Pratama, D. Wang, Deep stacked stochastic configuration networks for lifelong learning of non-stationary data streams, Information Sciences 495 (2019) 150-174.

[22] S. Liu, Q. Qu, S. Wang, Heterogeneous anomaly detection in social diffusion with discriminative feature discovery, Information Sciences 439 (2018) 118.

[23] https://www.theverge.com/2018/8/21/17763886/facebook-trustratings-fake-news-reporting-score

[24] https://www.engadget.com/2018/08/21/facebook-rates-usertrustworthiness/.

[25] C. Eiras-Franco, D. Martinez-Rego, B. Guijarro-Berdinas, A. AlonsoBetanzos, A. Bahamonde, Large scale anomaly detection in mixed numerical and categorical input spaces, Information Sciences 487 (2019) 115-127.

[26] C. Shi, Y. Li, J. Zhang, Y. Sun, S. Y. Philip, A survey of heterogeneous information network analysis, TKDE 29 (1) (2017) 17-37.

[27] S. Har-Peled, P. Indyk, R. Motwani, Approximate nearest neighbor: Towards removing the curse of dimensionality, Theory of computing 8 (1) (2012) 321-350.

[28] A. Gionis, P. Indyk, R. Motwani, et al., Similarity search in high dimensions via hashing, in: Vldb, Vol. 99, 1999, pp. 518-529. 
[29] M. S. Charikar, Similarity estimation techniques from rounding algorithms, in: Proceedings of the thiry-fourth annual ACM symposium on Theory of computing, 2002, pp. 380-388.

[30] S. Bhatia, B. Hooi, M. Yoon, K. Shin, C. Faloutsos, Midas: Microclusterbased detector of anomalies in edge streams., in: AAAI, 2020, pp. 32423249 .

[31] S. Ramaswamy, R. Rastogi, K. Shim, Efficient algorithms for mining outliers from large data sets, in: Proceedings of the 2000 ACM SIGMOD international conference on Management of data, 2000, pp. 427-438.

[32] C. Luo, A. Shrivastava, Ssh (sketch, shingle, \& hash) for indexing massivescale time series, in: NIPS 2016 Time Series Workshop, 2017, pp. 38-58.

[33] M. X. Goemans, D. P. Williamson, . 879-approximation algorithms for max cut and max 2sat, in: Proceedings of the twenty-sixth annual ACM symposium on Theory of computing, 1994, pp. 422-431.

[34] G. Cormode, S. Muthukrishnan, An improved data stream summary: The count-min sketch and its applications, in: latin american symposium on theoretical informatics, Springer, 2004, pp. 29-38.

995 [35] C. Luo, A. Shrivastava, Arrays of (locality-sensitive) count estimators (ace) anomaly detection on the edge, in: Proceedings of the 2018 World Wide Web Conference, 2018, pp. 1439-1448.

[36] M. Kulldorff, A spatial scan statistic, Communications in Statistics-Theory and methods (1997) 1481-1496.

[37] R. H. Berk, D. H. Jones, Goodness-of-fit test statistics that dominate the kolmogorov statistics, Probability theory and related fields (1979) 47-59.

[38] E. Álvarez-Miranda, I. Ljubić, P. Mutzel, The maximum weight connected subgraph problem, in: Facets of Combinatorial Optimization, 2013, pp. 245-270. 
[39] S. G. Santos, R. S. Barros, P. M. Gonçalves Jr, A differential evolution based method for tuning concept drift detectors in data streams, Information Sciences 485 (2019) 376-393.

[40] S. Kwon, M. Cha, K. Jung, Rumor detection over varying time windows, PloS one $12(1)$.

[41] D. Ge, X.-J. Zeng, Learning data streams online - an evolving fuzzy system approach with self-learning/adaptive thresholds, Information Sciences 507 (2020) $172-184$.

[42] S. Wasserman, K. Faust, et al., Social network analysis: Methods and applications, Vol. 8, Cambridge university press, 1994.

[43] D. J. Watts, S. H. Strogatz, Collective dynamics of 'small-world' networks, nature 393 (6684) (1998) 440-442.

[44] S. Speakman, Y. Zhang, D. B. Neill, Dynamic pattern detection with temporal consistency and connectivity constraints, in: 2013 IEEE 13th International Conference on Data Mining, IEEE, 2013, pp. 697-706.

[45] C. Castillo, M. Mendoza, B. Poblete, Information credibility on twitter, in: Proceedings of the 20th international conference on World wide web, 2011, pp. $675-684$.

[46] F. Yang, Y. Liu, X. Yu, M. Yang, Automatic detection of rumor on sina weibo, in: Proceedings of the ACM SIGKDD Workshop on Mining Data Semantics, 2012, pp. 1-7.

[47] G. Cormode, S. Muthukrishnan, An improved data stream summary: the count-min sketch and its applications, Journal of Algorithms 55 (1) (2005) $58-75$.

[48] A. Olteanu, C. Castillo, F. Diaz, S. Vieweg, Crisislex: A lexicon for collecting and filtering microblogged communications in crises, in: ICWSM, 2014, pp. 376-385. 
[49] N. Pham, R. Pagh, A near-linear time approximation algorithm for anglebased outlier detection in high-dimensional data, in: Proceedings of the 18th ACM SIGKDD international conference on Knowledge discovery and data mining, 2012, pp. 877-885.

[50] D. J. Hand, N. M. Adams, Data mining, Wiley StatsRef: Statistics Reference Online (2014) 1-7. 